\title{
1 Direct Simulation of Conjugate Heat 2 Transfer of Jet in Channel Crossflow
}

3 Z. $\mathrm{Wu}^{1 *}$, D. Laurence ${ }^{1}, \mathrm{H}$. Iacovides ${ }^{1}$ and I. Afgan ${ }^{1}$

$4{ }^{1}$ Modelling and Simulation Centre, School of Mechanical, Aerospace and Civil Engineering,

5 The University of Manchester

6 Oxford Road, Manchester M13 9PL, UK, zhao.wu@ manchester.ac.uk

7 Key words: jet in channel crossflow; conjugate heat transfer; direct numerical simulation; low

8 jet-to-crossflow velocity ratio

9 Abstract: We present a DNS study of a hot, low momentum laminar water jet discharged into a cold turbulent channel stream through a circular orifice in one of the steel channel walls. The channel wall has a finite thickness and its outer side is cooled under Robin type thermal boundary conditions for a realistic external environment, leading to a conjugate heat transfer system. Nusselt number and r.m.s temperature fluctuations on the wall are compared with our earlier DNS results for the simpler iso-thermal and adiabatic conditions at the channel inner surface. Temperature fluctuations inside the channel wall are resolved to provide data for a conjugate heat transfer ( $\mathrm{CHT})$ thermal fatigue test case related to the ageing of pipe walls and welds studies, as found, for example, in power plant piping Tjunctions. The crossflow Reynolds number is $R e=3333$, jet-to-crossflow velocity ratio is $R=1 / 6$ and fluid-to-solid conductivity ratio is $1 / 64$.

The near-wall mean flow structures, a horseshoe vortex ahead and on the sides of the jet orifice, a shallow recirculation behind the discharge and a counter-rotating vortex pair drawing in a blanket of cooler cross-flow, lead to a complex convective and turbulent wall heat transfer pattern around the orifice. The main findings are:

(i) Wall maps of Nusselt number and r.m.s temperature, $\theta_{\text {r.m.s }}$, for conjugate heat transfer

(ii) Inside the solid $\theta_{\text {r.m.s }}$ and its dissipation, analysed from RANS modelling perspective, show that predicted thermal spot length scales are discontinuous on the interface, at variance with the 2-point spectrum-derived scales.

(iii) At the high wavenumber range, the spanwise temperature spectra decrease according to exponential-decay spectral models for the fluid turbulence in the Kolmogorov range, but with large exponential coefficients increasing with depth inside the solid. 


\section{Nomenclature}

$\begin{array}{clll}C_{1}, C_{2} & \text { constants } & v & \text { kinematic viscosity } \\ c_{p} & \text { specific heat } & \rho & \text { density } \\ E & \text { spectrum } & \Omega & \text { volume domain } \\ G & \alpha_{f} / \alpha_{s} \text { ratio of thermal } & & \\ & \text { diffusivities } & & \\ h & \text { convective heat transfer }\end{array}$$$
\text { coefficient }
$$

$H \quad$ half of channel height and

Subscripts

radius of jet-exit

$k \quad$ turbulent kinetic energy

$(\cdot)_{c} \quad$ crossflow

$\mathrm{Nu} \quad$ Nusselt number

Pr Prandtl number

r.m.s root-mean-square

$R$

$\left\langle u_{j}\right\rangle /\left\langle u_{c}\right\rangle$ jet-to-crossflow

velocity ratio

Re Reynolds number

$t \quad$ time

$T \quad$ temperature

$u, v, w \quad$ velocity components in $x, y$

and $z$ directions

$x \quad$ streamwise distance

$y \quad$ wall-normal distance

$z \quad$ spanwise distance

Greek symbols

$\begin{array}{cl}\alpha & \lambda / \rho c_{p} \text { thermal diffusivity } \\ \beta_{1}, \beta_{2} & \text { constants } \\ \Gamma & \text { fluid-solid interface } \\ \varepsilon & \text { dissipation rate } \\ \eta & \text { Kolmogorov length scale } \\ \theta & \left(T-T_{c}\right) /\left(T_{j}-T_{c}\right) \\ & \text { dimensionless temperature } \\ \kappa & \text { wavenumber } \\ \lambda & \\ & \text { thermal conductivity }\end{array}$

Abbrev.

CHT conjugate heat transfer

DNS direct numerical simulation

JICF jet in crossflow

LES large-eddy simulation

PWR pressurised water reactor

RANS Reynolds-averaged Navier-

Stokes

1

\section{Introduction}

3 Jets in crossflow (JICF) are of great industrial interest with applications in turbine blades film

4 cooling, de-icing of aeroplane wings, power plant piping systems and pollutant into water or

5 atmosphere. Many studies on this type of problem have been motivated and reported, either

6 experimental [1-9] or numerical [10-14]. 


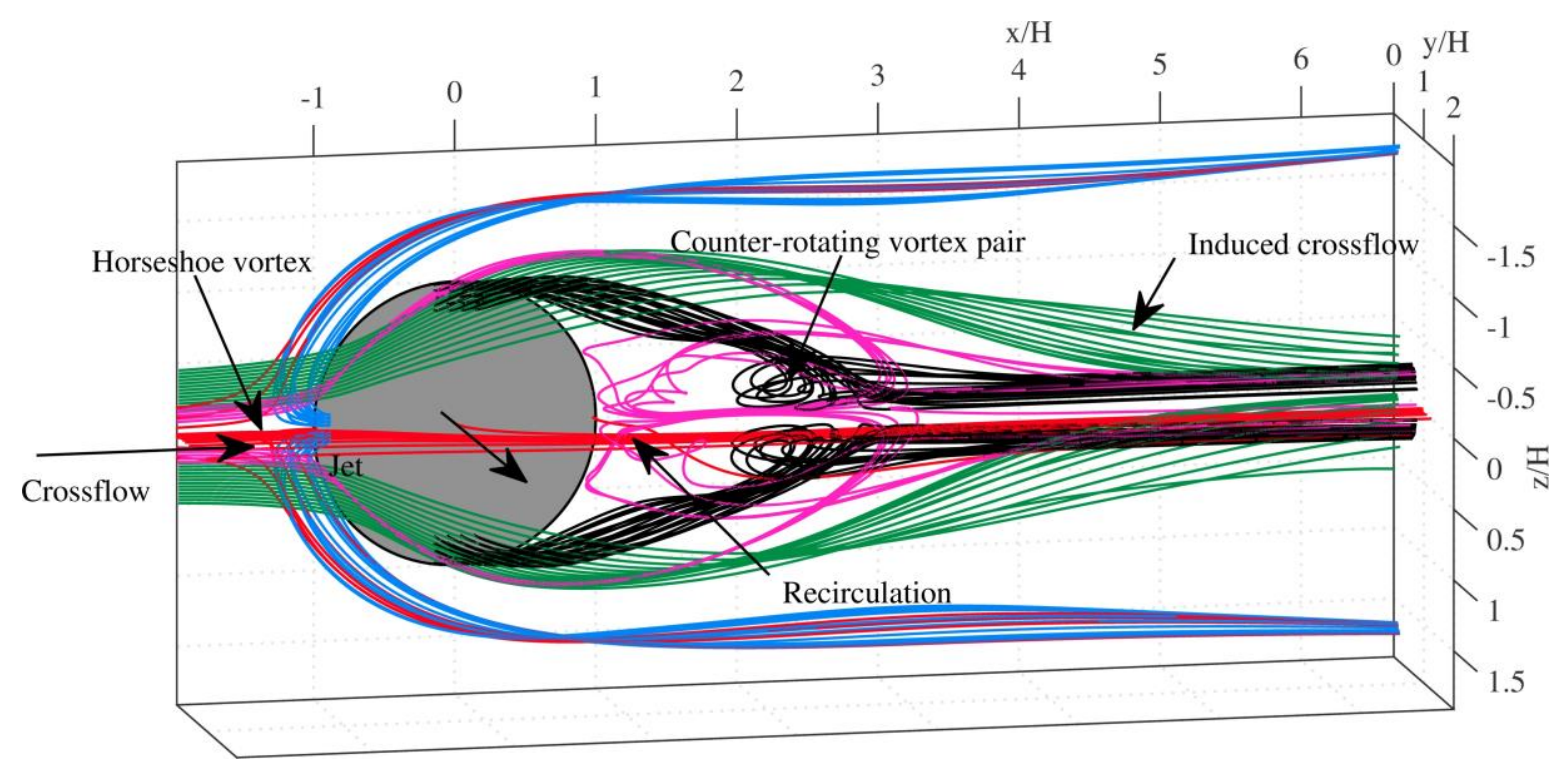

(a)

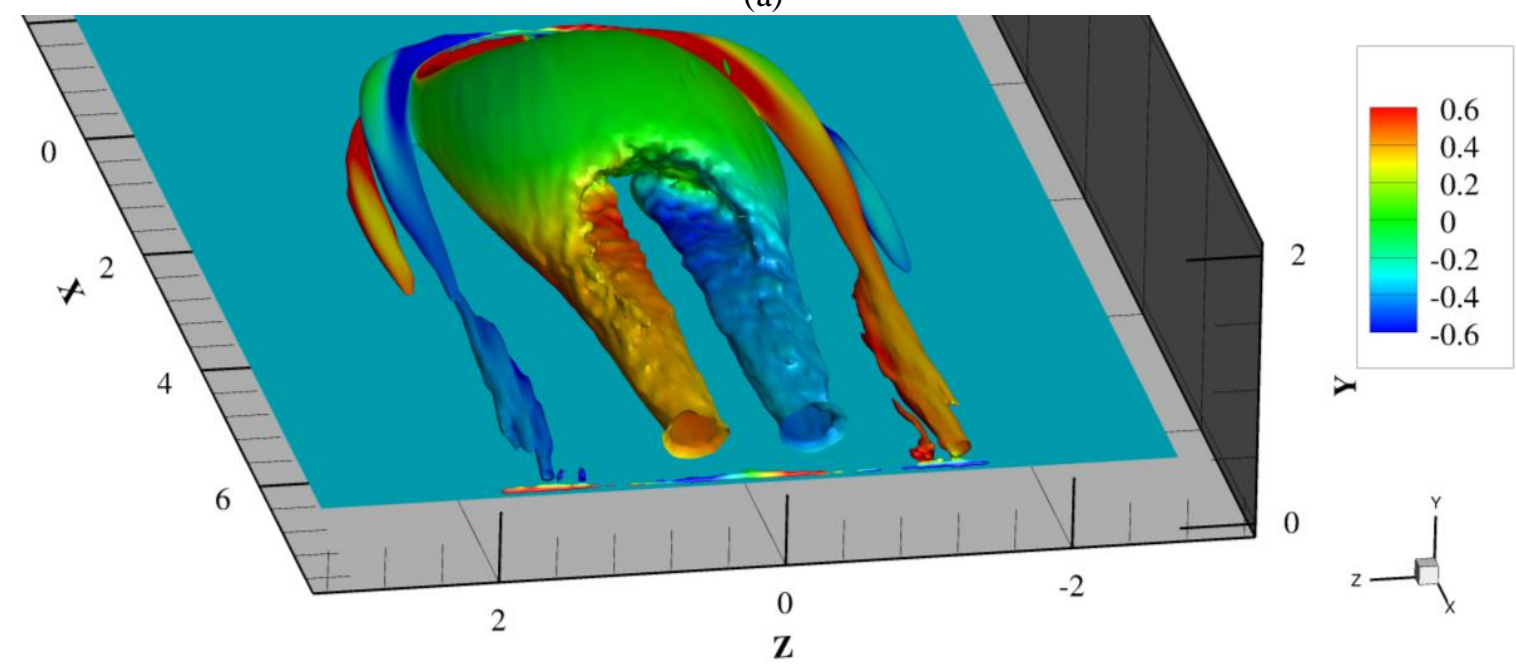

(b)

Figure 1. Structures of jet in crossflow with a low velocity ratio: (a) time-averaged streamline; (b) iso-surface of Q-criterion $(Q=0.5)$ coloured by the streamwise vorticity $\Omega_{x}$.

1 However, few studies $[11,15,16]$ are focused on the jet in crossflow problems with low jet-to-

2 crossflow velocity ratios, $R$, which occur e.g. in T-junction pipes of a nuclear power plant

3 emergency cooling systems. In a jet in crossflow with low jet-to-crossflow velocity ratios,

4 there are three dominant time-averaged vortex structures (Figure 1): a horseshoe vortex at the

5 front edge of jet-exit, a counter-rotating vortex pair below the non-uniform velocity jet and a

6 recirculation in the wake region [17].

7 Temperature differences between two piping flow streams will result in temperature

8 fluctuations in the piping wall and may lead to thermal striping: high cycle thermal stress

9 induced wall weakening, and possibly failure [18], particularly for the low momentum jet in

10 crossflow where the secondary injection remains close to the T-junction welds. Prediction of

11 thermal fatigue in a nuclear power plant piping system is a complex material science ageing 
1 problem but it is already challenging from the more limited thermal loading aspect studied herein. Perhaps the French Civaux pressurised water nuclear reactor (PWR) plant in 1998 [19] and the Japanese Tsuruga-2 and Tomari-2 PWR plants in 1999 and 2003 [20] are the most notable among this type of safety incidents.

5 To predict thermal fatigue, one needs to identify the temperature fluctuation magnitudes and 6 frequencies inside the solid piping wall. It requires the coupling of heat transfer between fluid and structure, called conjugate heat transfer (CHT) analysis. The numerical simulations of conjugate heat transfer of a jet in crossflow at present are mostly based on Reynolds-averaged or large-eddy methods, and their reliability needs further validation. Experimental data on the conjugate heat transfer is rare as walls in lab rigs are usually adiabatic, since the transparent Plexiglas is often the material used, which allows optical access for flow and turbulence characteristics measurements. Thus, the present study objective is to generate a reliable and comprehensive DNS database for jets in channel crossflow at a low jet-to-crossflow velocity ratio, corresponding to a real industrial situation, but for which no experimental data is available. Both time-averaged and instantaneous profiles are examined, to understand the physics of jet in crossflow at low jet-to-crossflow velocity ratios and the conjugate heat transfer problem. The data can also help test and improve the engineering RANS and LES turbulence models, which so far mostly relied on simple channel flow DNS, such as reference [21]. Our earlier DNS results for adiabatic and the iso-thermal thermal boundary conditions applied to the fluid domain [22] are included here for comparison to evaluate the relevance of these simpler test case conditions. In this series study, we described the flow physics of the jet in crossflow in [17], used proper orthogonal decomposition and dynamic mode decomposition to study the coherent structures in [23] and evaluated the RANS models in these simple cases in [24]. The database is accessible free online (http://dx.doi.org/10.17632/7nx4prgizz.2) [25].

Section 2 provides the numerical details of conjugate heat transfer extension, followed by the results and discussions in Section 3 and conclusions in Section 4.

\section{Numerical details of conjugate heat transfer}

The DNS simulations are performed with the computational fluid dynamics code

30 Incompact3d; a highly accurate and widely benchmarked open-source DNS code [26,27].

31 More than 40 papers have been published, based on simulations arising from the use of this code (cf. http://www.incompact3d.com/). Recently Flageul et al. [28] equipped Incompact3d 
1 with the ability to solve conjugate heat transfer in periodic channel flows. These developments were validated against analytical solutions and rarer DNS data of channel flow with conjugate heat transfer. The authors in present paper extended their code for a 3-D jet in channel crossflow configuration.

\section{$5 \quad 2.1$ Numerical methods}

6 The present DNS of the jet in crossflow employed the same numerical scheme as that in [17] in the fluid domain, a sixth-order central compact finite scheme applied to the velocity components stored on a collocated grid and a spectral method for solving the pressure Poisson equation on a staggered grid. The second-order Adams-Bashforth scheme is used for the time-advancement.

The numerical method in the solid domain and the fluid-solid coupling are described in [28] and are available as an open-source module with minimal changes to the code. This section summarises numerical methods used in the solid domain and fluid-solid coupling, as well as those modifications made to Flageul's code. Hereafter, the subscript "s" denotes the solid, while "f" denotes the fluid.

Thermal conduction in the solid is governed by the following differential equation:

$$
\partial_{t} T_{s}=\alpha_{s}\left(\nabla_{\mathrm{x}}^{2} T_{s}+\nabla_{\mathrm{y}}^{2} T_{s}+\nabla_{\mathrm{z}}^{2} T_{s}\right)
$$

17 where $T_{s}$ and $\alpha_{s}=\lambda_{s} /\left(\rho_{s} c_{p, s}\right)$ are the solid temperature and thermal diffusivity, with $\lambda_{s}, \rho_{s}$ and $c_{p, s}$ the solid thermal conductivity, density and specific heat capacity respectively. For simplicity " $x$ " and " $z$ " denote the main channel streamwise and spanwise wall parallel axes, and " $y$ " the wall normal one.

The numerical implementation of the solid heat transfer in the wall-parallel directions and the wall-normal one are different. In the wall-parallel directions, the solid domain shares the same Cartesian grid coordinate system as the fluid one: the nodes are equally spaced in $x$ and $z$ directions. The $x$ and $z$ diffusive terms are computed using the finite difference method. The second-order central difference scheme (one-sided scheme at the boundary) is applied to the wall parallel diffusive terms in the solid, instead of the sixth-order one for the diffusive terms in the fluid domain. This change reduced the computational efforts with the added benefit of easily handling the non-periodic boundary conditions in $x$ directions which are not present in 
1 differencing entails some loss of accuracy but only at high wavenumbers [29] and profiles are obviously smoother in the solid, especially in wall-parallel directions.

3 In the wall-normal direction, the solid domain is discretised with $n$ Chebyshev nodes. This 4 makes the grid suitably refined at the wall to catch near-wall phenomenon, as the normal 5 temperature gradient is large in the region near the fluid-solid interface. The temperature and 6 its wall normal derivatives are obtained using Lagrange interpolation polynomials. For the time-advancement of Eq. (1), a second-order Adams-Bashforth scheme is applied to the wallparallel terms but a backward Euler one for the wall-normal component.

9 Conjugate heat transfer couples the fluid and solid thermal conditions: the temperature and 10 heat flux are continuous at the interface. Therefore, the conjugate heat transfer problems can 11 be described as follows:

$$
\begin{gathered}
\partial_{t} T_{f}+\partial_{i}\left(T_{f} u_{i}\right)=\alpha_{f} \nabla^{2} T_{f} \text { in the fluid domain } \Omega_{f} \\
\partial_{t} T_{s}=\alpha_{s} \nabla^{2} T_{s} \text { in the solid domain } \Omega_{s} \\
T_{s}=T_{f} \text { on the fluid-solid interface } \Gamma \\
\partial_{n} T_{s}=\frac{\lambda_{f}}{\lambda_{s}} \partial_{n} T_{f} \text { on the fluid-solid interface } \Gamma
\end{gathered}
$$
where $\alpha_{f} / \alpha_{s} \equiv G$ is the ratio of fluid to solid thermal diffusivity, $\Omega_{f}$ and $\Omega_{s}$ the fluid and solid domains, and $\Gamma$ the fluid-solid interface on which $\partial_{n} T$ is highly discontinuous since $\lambda_{f} / \lambda_{s} \ll 1$. One way of solving the above equations is to set up a tightly coupled discretization in both the fluid and solid domains which removes stability issues associated with the interface characteristics [30,31]. Nevertheless, it would be difficult to implement as the governing equations and the discretization methods in solid and fluid are often different and the temperature gradient is discontinuous.

Giles [30] also suggested an alternative loosely coupled method that overcomes this difficulty but maintains stability in most fluid-solid interaction cases. At time-step $n$, both $T_{s}$ and $T_{f}$ are available. At the next time-step $n+1$, the fluid temperature is updated using the new fluid velocity field with a Dirichlet boundary condition: $T_{f}^{n+1}=0.5\left(T_{f}^{n}+T_{s}^{n}\right)$ on $\Gamma$. Then the solid temperature is updated with a Neumann boundary condition: $\partial_{n} T_{s}^{n+1}=\frac{\lambda_{f}}{\lambda_{s}} \partial_{n} T_{f}^{n+1}$ at the interface. As a result, at the end of the new time-step, the heat flux at the interface is continuous, while the temperature at the interface is expected to have a first-order error in 
1 time [32] which is not an issue since a small time-step is imposed by DNS of wall turbulence 2 requirements. Further details can be found in [17,33].

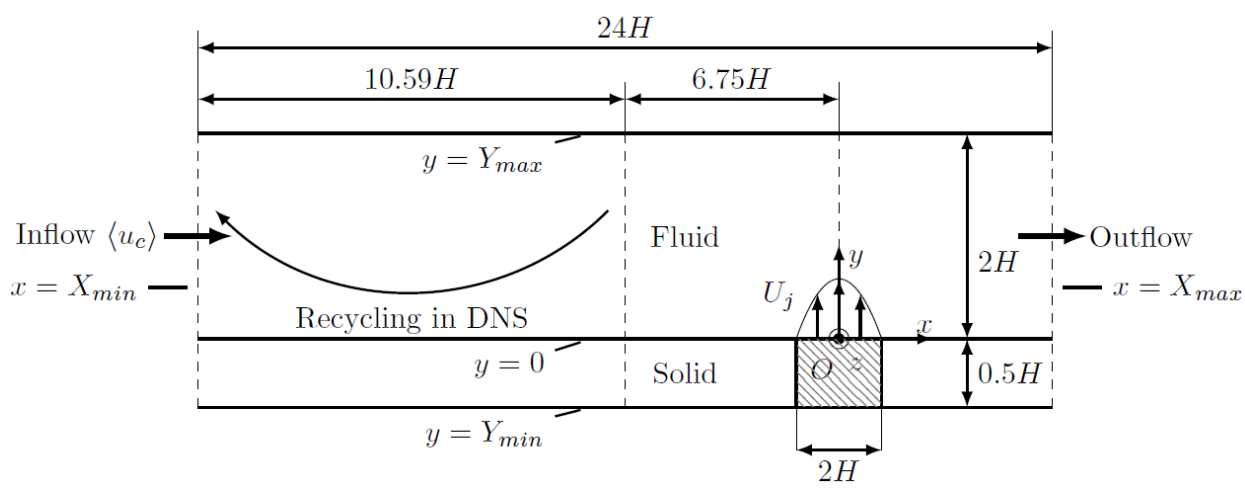

(a)

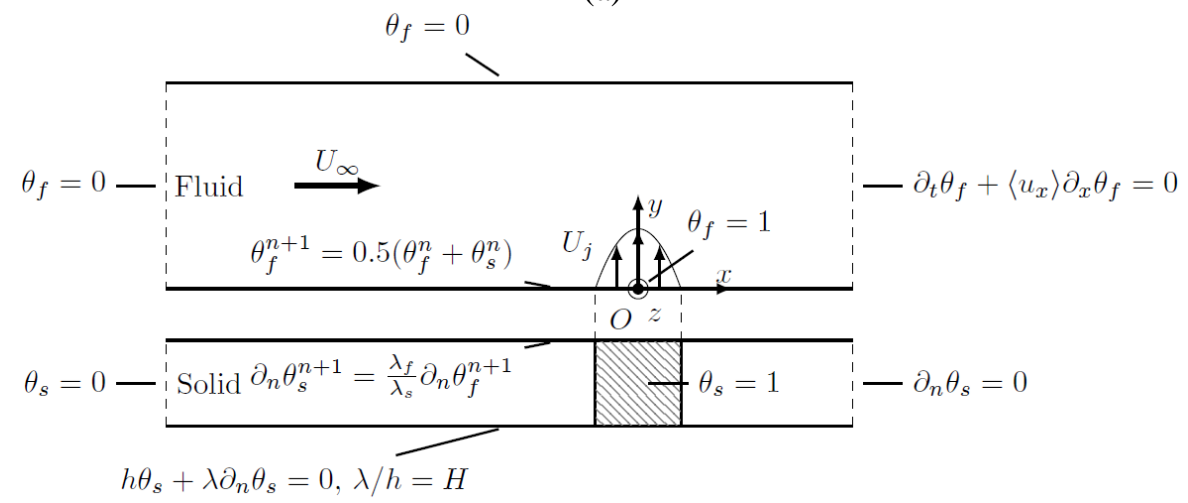

(b)

Figure 2. (a) Schematic view of the flow configuration and (b) thermal boundary conditions of the present jet in channel flow DNS. The origin point is located at the centre of jet-exit. A recycling method is used before the jet to generate a fully turbulent inflow. The temperature of shaded area in solid is set at $\theta_{s}=1$. Figures are not drawn to scale.

\section{$3 \quad 2.2$ Simulation configurations}

4 Figure 2(a) shows the flow configuration of the current simulation. The fluid domain is 5 chosen to correspond to the DNS study of [17], i.e. $[-17.3 H, 0 H,-3 H] \leq[x, y, z] \leq$ $6 \quad[6.7 H, 2 H, 3 H]$. The crossflow Reynolds number is set at $\operatorname{Re}=\left\langle u_{c}\right\rangle H / v=3333$, where $\left\langle u_{c}\right\rangle$

7 is the bulk crossflow velocity, $v$ the fluid kinematic viscosity. The jet-to-crossflow velocity 8 ratio $R$ is set at $\left\langle u_{j}\right\rangle /\left\langle u_{c}\right\rangle=1 / 6$. The jet-exit with a diameter equal to the channel height $2 H$,

9 is located at origin point.

10 The Prandtl number is set at $\operatorname{Pr}=0.7$. The temperature is normalised by the jet and crossflow 11 temperature differences, i.e. $\theta=\left(T-T_{c}\right) /\left(T_{j}-T_{c}\right)$, leading to the non-dimensional 12 temperature of unity at jet inlet but zero in crossflow inlet. The ratio of thermal diffusivity $13 G=\alpha_{f} / \alpha_{s}$ is set as $0.0172 \sim 1 / 58$ and the ratio of thermal conductivity as $\lambda_{f} / \lambda_{s}=$ 
$10.0156 \sim 1 / 64$. These values typically represent the classical conditions in a pressurized water nuclear reactor (PWR).

3 No-slip wall boundary conditions are applied at the top and bottom fluid domain. Periodic 4 boundary conditions are applied to both the fluid and solid spanwise planes. An interior recycling method [34] is used in the upstream part of the channel to provide fully-turbulent crossflow profiles before the jet-exit. The corresponding Reynolds number based on wall shear stress, $\operatorname{Re}_{\tau}=\left\langle u_{\tau}\right\rangle H / v$, is 208 in the present study, where $\left\langle u_{\tau}\right\rangle$ is the averaged friction velocity within the recycling region. The velocity profile at the jet inlet is assumed to be parabolic, implying laminar fully developed conditions. This jet profile set-up, similar to several other numerical works [35-39], provides a better defined inlet condition which makes the case easy for CFD validation, although the configuration leads to a purely numerical experiment. At the outlet section the simple convective outflow boundary conditions [40] are prescribed.

The thermal boundary conditions are shown in Figure 2(b). As the effect of hot jet on the top wall is negligible [17], only the solid domain below the fluid domain $(-0.5 H \leq y \leq 0 H)$ is modelled to save computational resources, and the iso-thermal boundary condition is applied on the fluid top wall, i.e. $\left.\theta_{f}\right|_{y=Y_{\max }}=0$. This solid domain is discretized with 513/256 equalspaced Cartesian nodes in $x / z$ directions which share the same coordinates as the fluid domain mesh, but with $97 \mathrm{Chebyshev}$ nodes in the $y$ direction. As a result, the mesh step $\Delta y$ at the interface is $3.3 \times 10^{-5} \mathrm{H}$ on the solid side and $0.033 \mathrm{H}$ on the fluid side. The round jet-exit is finely pixeled (see Figure 1 in [17]) in the open-source DNS code, Incompact3d, which has been similarly applied to many curved surfaces (round impinging jet [41], leading edge curvature effects [42], flow past a cylinder [43] to name a few). The boundary temperature at the solid wall under the fluid inlet is set as $\left.\theta_{S}\right|_{x=X_{\min }}=0$. In the solid region under the jetexit $\left(x^{2}+z^{2} \leq 1, y<0\right.$, the shaded area in Figure 2), the uniform temperature $\theta_{s}=1$ is imposed all the time to mimic the injection pipe and acts as the boundary for the heat equation. A classical Robin boundary condition is set at the outer surface of solid wall, i.e. $\left.\left(h \theta_{s}+\lambda \partial_{n} \theta_{s}\right)\right|_{y=Y_{\min }}=0$, with $\lambda / h=H$ (meters). This Robin boundary condition represents modelling of heat losses at the solid outer surface with the transfer coefficient $h$. Herein $H=1 \mathrm{~m}$ was chosen.

In addition to the conjugate heat transfer case, two non-conjugate heat transfer cases were also simulated. In these two cases, the solid domain was omitted, and the adiabatic $\left(\partial_{n} \theta_{f}=0\right)$ 
1 or iso-thermal $\left(\theta_{f}=0\right)$ condition were directly imposed to the fluid top $\left(y=Y_{\max }\right)$ and 2 bottom $(y=0)$ boundaries.

3 The time-step of the current simulation is fixed at $\Delta t=6 \times 10^{-4} H /\left\langle u_{c}\right\rangle$. The discretized 4 instantaneous flow equations are solved first over the fluid domain, decoupled from the energy equation. The time-marching solution of the energy equation starts once the timemarching solution of the flow field advances enough for the predicted flow to become fully turbulent. The temperature in both fluid and solid domain is initially set to zero. The initial transient lasts for $360 \mathrm{H} /\left\langle u_{c}\right\rangle$ simulation time units. The time-averaged profiles have been obtained by averaging the instantaneous solution at every time-steps during the simulation after the transition. The mean profiles are averaged over $5940 \mathrm{H} /\left\langle u_{c}\right\rangle$ simulation time units equal to 247.5 whole channel flow-through time. Further details, including the grid resolution study and the validation of the inflow turbulence recycling method, can be found in [17].

The present DNS simulations have been performed on the EDF R\&D's BlueGene/Q highperformance computing facility, with 4096 CPU cores. Each time-step took 0.151s wall-clock time, about $10 \%$ of which was devoted to solving solid thermal equations.

\section{Results and discussions}

\subsection{Time-averaged profiles}

Time-averaged contours at the fluid-solid interface in the conjugate heat transfer, adiabatic and iso-thermal cases are presented in Figure 3 to 7. The non-dimensional mean wall temperature $\langle\theta\rangle_{\text {wall }}$ and its root-mean-square (r.m.s) $\theta_{\text {r.m.s }}$ in the iso-thermal case, as well as the mean Nusselt $(\mathrm{Nu})$ number in the adiabatic case, are zero on the interface, and therefore omitted.

Figure 3 and 4 show the mean wall temperature and Nusselt number, respectively. On the wall surface, the impact of the hot jet fluid at $x / H<0$ is limited to a small region in the adiabatic and iso-thermal cases due to the high-speed cold crossflow. However, in the conjugate heat transfer case, the solid is heated by the hot injection pipe leading to an almost concentric temperature profile around the jet-exit (left of Figure 3). Upstream of the injection, $x / H<0$, negative Nusselt number values are found since the wall temperature is higher than that of the fluid stream (left of Figure 4). On the contrary, in the wake region, where the hot jet and cold crossflow mix, thermal energy is transferred from the fluid to the wall. 
1 Note that the mean temperature in the adiabatic case and the Nusselt number in the isothermal case have a similar pattern (right of Figure 3 and 4), whereas in the conjugate heat transfer case they are very different. In the latter case, three phenomena overlap: isotropic conduction in the steel wall, classical convective heat transfer upstream of the jet inlet, and finally mostly turbulent heat transfer downstream.

6 On the other hand, the non-dimensional $\theta_{\text {r.m.s }}$ distributions on the fluid-solid interface for the conjugate heat transfer and adiabatic cases shown in Figure 5, now with each case in it is own colour scaling, are similar in pattern but not in magnitude. The interface $\theta_{\text {r.m.s }}$ in conjugate heat transfer is much lower than that in the adiabatic case; the maximum interface $\theta_{\text {r.m.s }}$ in the conjugate heat transfer case is about $1 / 20$ of the adiabatic one. This is due to the high heat conduction in the solid that smooths out rapidly small-scale and high frequency temperature fluctuations even on the surface. Despite this, both cases show a qualitatively similar pattern. High interface $\theta_{\text {r.m.s }}$ legs rounding the jet-exit indicate the heat mixing between the jet and crossflow fluid is strong there, as explained in [22], where it was found that $\theta_{\text {r.m.s }}$ has a strong correlation to mean turbulence kinetic energy $\langle k\rangle$.

Figure 6 shows the mean temperature $\langle\theta\rangle$ on over the symmetry plane. The heat conduction in the solid is obvious near the injection pipe in the conjugate heat transfer case. For $x / H<0$, upstream of the injection, the cold fluid above the solid is heated by the wall as per the negative $\mathrm{Nu}$ number shown in Figure 4. The fluid temperature of the conjugate heat transfer case is somewhat better approached by the adiabatic case in the near wake region, but further downstream the iso-thermal case is closer. In the adiabatic case, the boundary condition $\partial \theta / \partial y=0$ is only seen in a very thin layer on the wall (also see Figure 11 of [17]) as the hot fluid from the two sides of jet-exit (black streamlines in Figure 1(a)) sits on the wall and remains extremely low downstream. In some applications, e.g. turbine film cooling and aeroplane wing de-icing, this desirable feature helps protect the surface. However this kink in mean temperature profiles is smoothed out by wall-conduction in the conjugate heat transfer case.

Figure 7 shows the fluctuating temperature r.m.s, $\theta_{\text {r.m.s }}$, over the symmetry plane in logarithmic scale. On a log scale, the iso-thermal and adiabatic cases approach the $\theta_{\text {r.m.s }}$ variation of the conjugate heat transfer case somewhat. In this low-momentum and low-Re jet in channel crossflow case with high (steel) thermal conductivity, the fluid is seen to be heated up significantly upstream of the injection. There, where a weld might be, the fluctuations 
1 penetrate deep into the solid wall as they are mainly the low-frequency fluctuations [44]. On

2 the downstream side, low-frequency fluctuations deep inside the steel are also found further

3 away from the weld but this obviously depends on the chosen $R, \operatorname{Re}$ and $G$ values. Figure 8

4 shows the $\theta_{\text {r.m.s }}$ profiles at selected downstream locations. As the flow develops downstream,

5 the upper mixing shear layer rises and so does the peak of $\theta_{\text {r.m.s }}$ profiles on the symmetry

6 plane $(z / H=0)$ but the secondary peak below the jet remains very close to the wall. On the

7 plane
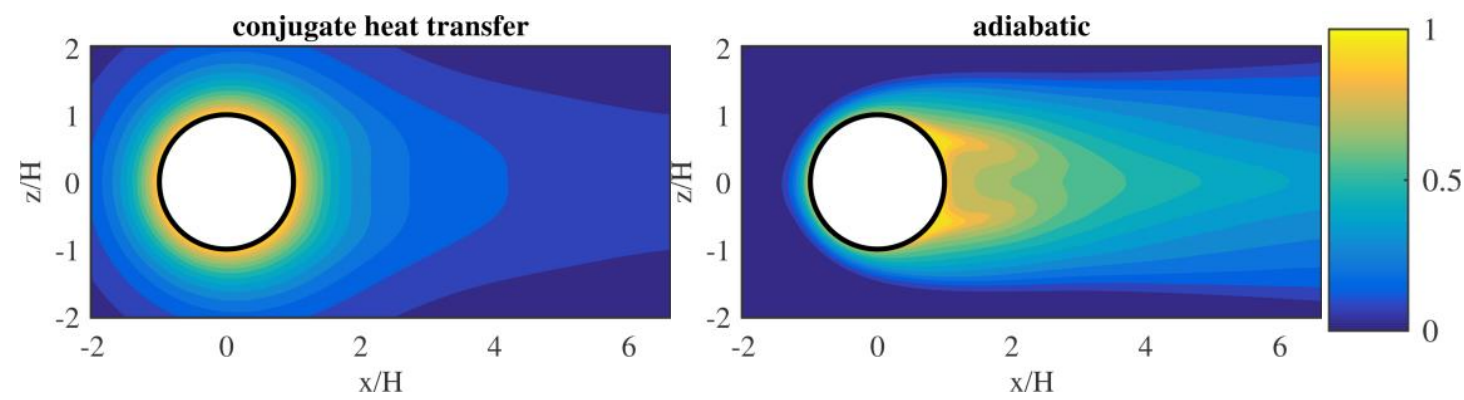

Figure 3. Non-dimensional mean wall temperature $\langle\theta\rangle_{\text {wall }}$ on the fluid-solid interface in conjugate heat transfer (left) and adiabatic (right) cases.
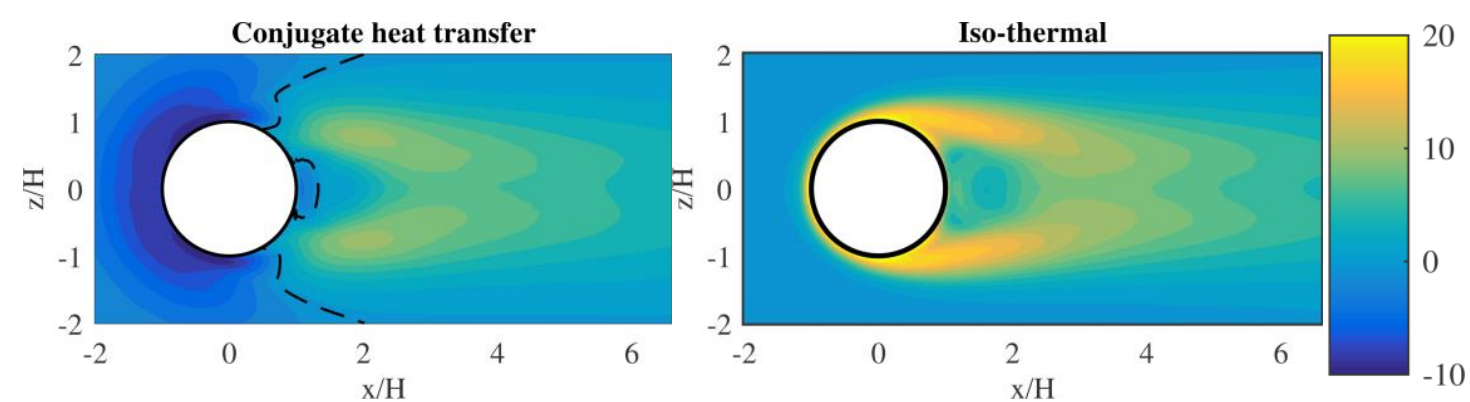

Figure 4. Mean Nusselt number $\mathrm{Nu}$ on the fluid-solid interface in conjugate heat transfer (left) and isothermal (right) cases. The dashed line indicates the $\mathrm{Nu}=0$ isoline. Negative $\mathrm{Nu}$ represents heat transfers from solid to fluid.
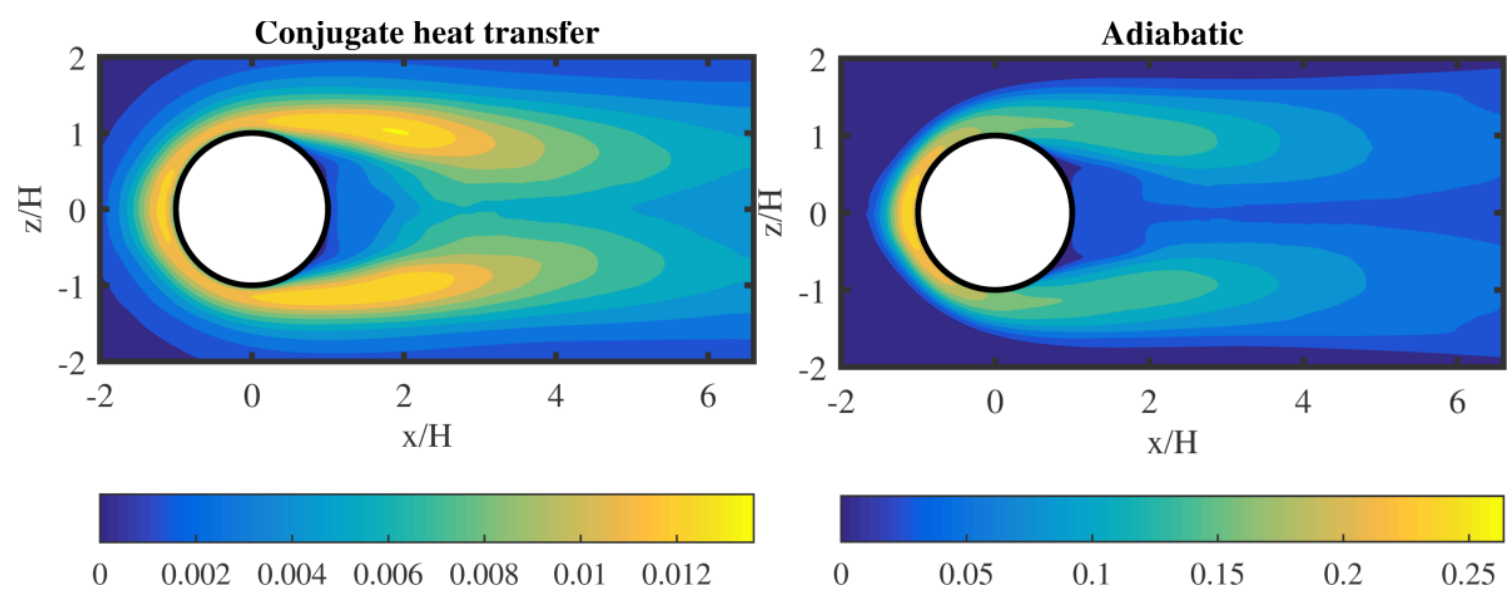

Figure 5. Non-dimensional temperature r.m.s $\theta_{\text {r.m.s }}$ on the fluid-solid interface in conjugate heat transfer (left) and adiabatic (right) cases. Plotted in their own scales. 
$1 z / H=1$ the peak in $\theta_{\text {r.m.s }}$ is close to the wall and just as intense as in the shear layer. In the 2 conjugate heat transfer case only the additional log scale allows to see deep penetration in the 3 solid, but above it in the laminar fluid layer, $\theta_{\text {r.m.s }}$ decays very fast close to the interface due 4 to discontinuity of thermal conductivity. The $\theta_{\text {r.m.s }}$ in the solid is about one order of magnitude lower than that in the fluid viscous sublayer. At most observation stations, the conjugate heat transfer $\theta_{\text {r.m.s }}$ profiles in the fluid are quite similar to the iso-thermal ones except upstream of jet $(x / H=-1, z / H=1)$.
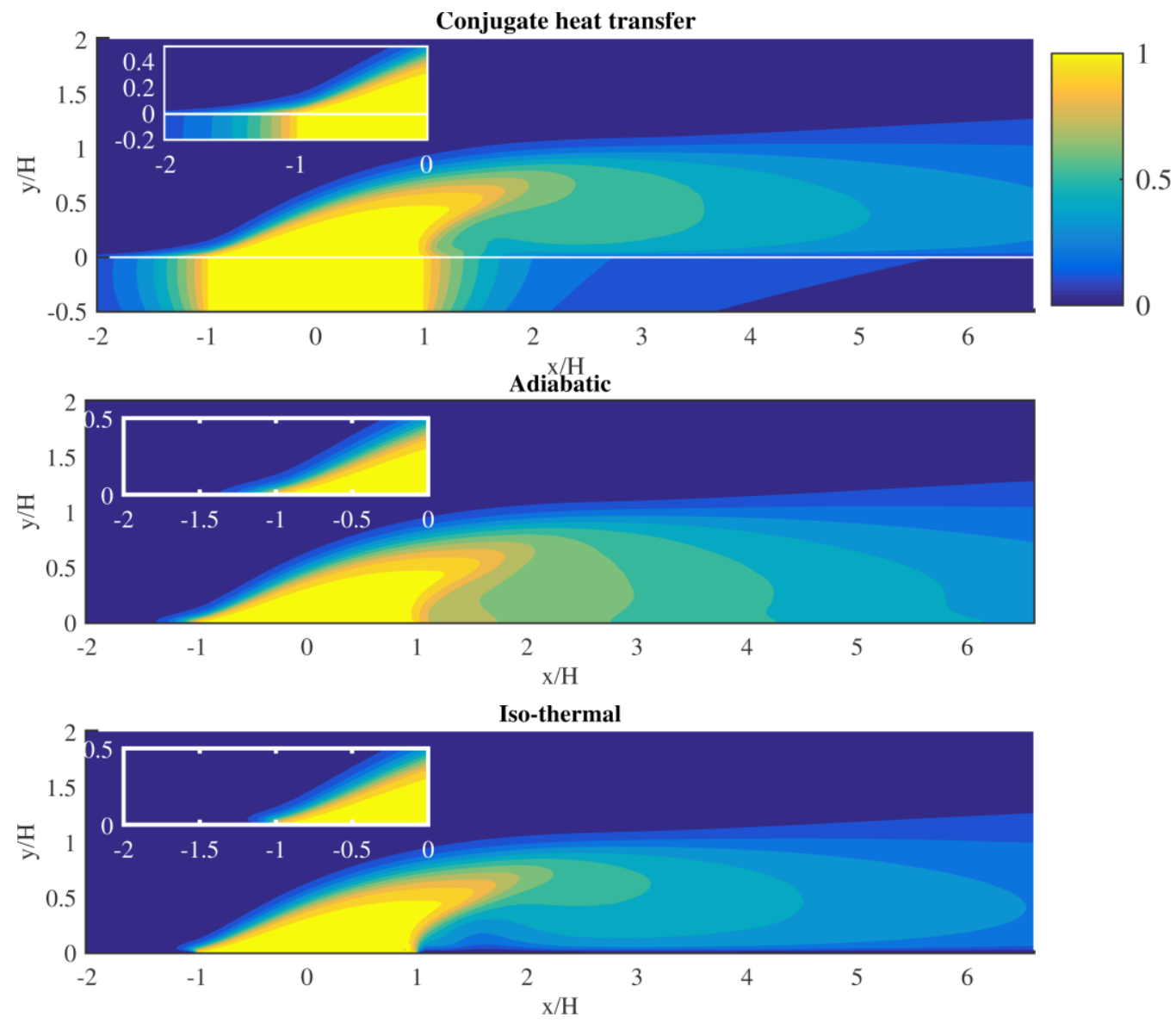

Figure 6. Non-dimensional mean temperature $\langle\theta\rangle$ on the symmetry plane in conjugate heat transfer, adiabatic and iso-thermal cases.

9 Figure 9 and 10 show the non-dimensional heat fluxes on the symmetry plane $(z / H=0)$ and the plane $z / H=1$ respectively. These heat fluxes are normalized here by $\left\langle u_{c}\right\rangle\left(\theta_{j}-\theta_{c}\right)$ as the wall friction velocity is highly variable. Eventually they could be normalized with the upstream channel friction $\left\langle u_{\tau_{c}}\right\rangle\left(\theta_{j}-\theta_{c}\right)$, where $\left\langle u_{\tau_{c}}\right\rangle /\left\langle u_{c}\right\rangle=0.0624$ in present study. The spanwise turbulent heat flux was checked to be null on $z / H=0$ due to symmetry, and thus is only presented on the plane $z / H=1$. The heat flux structures over the plane $z / H=1$ are nearer to the bottom wall compared to those over the symmetry plane, $z / H=0$, profiles. 
1 This is because the crossflow acts like a "partial cover" $[5,45,46]$ above the jet, and the shear 2 layer region is lower on the spanwise side (see Figure 1). The profiles for the three thermal 3 boundary condition cases show agreement in the region above the jet-crossflow shear layer, 4 but behave differently in the wake region. In the fluid, the heat flux profiles with the conjugate heat transfer again share some similarities with those for the iso-thermal condition, as seen in the heat flux profiles, except upstream of jet $(x / H=-1, z / H=1)$. However one should bear in mind that these similarities between the conjugate heat transfer and isothermal cases are only observed for the
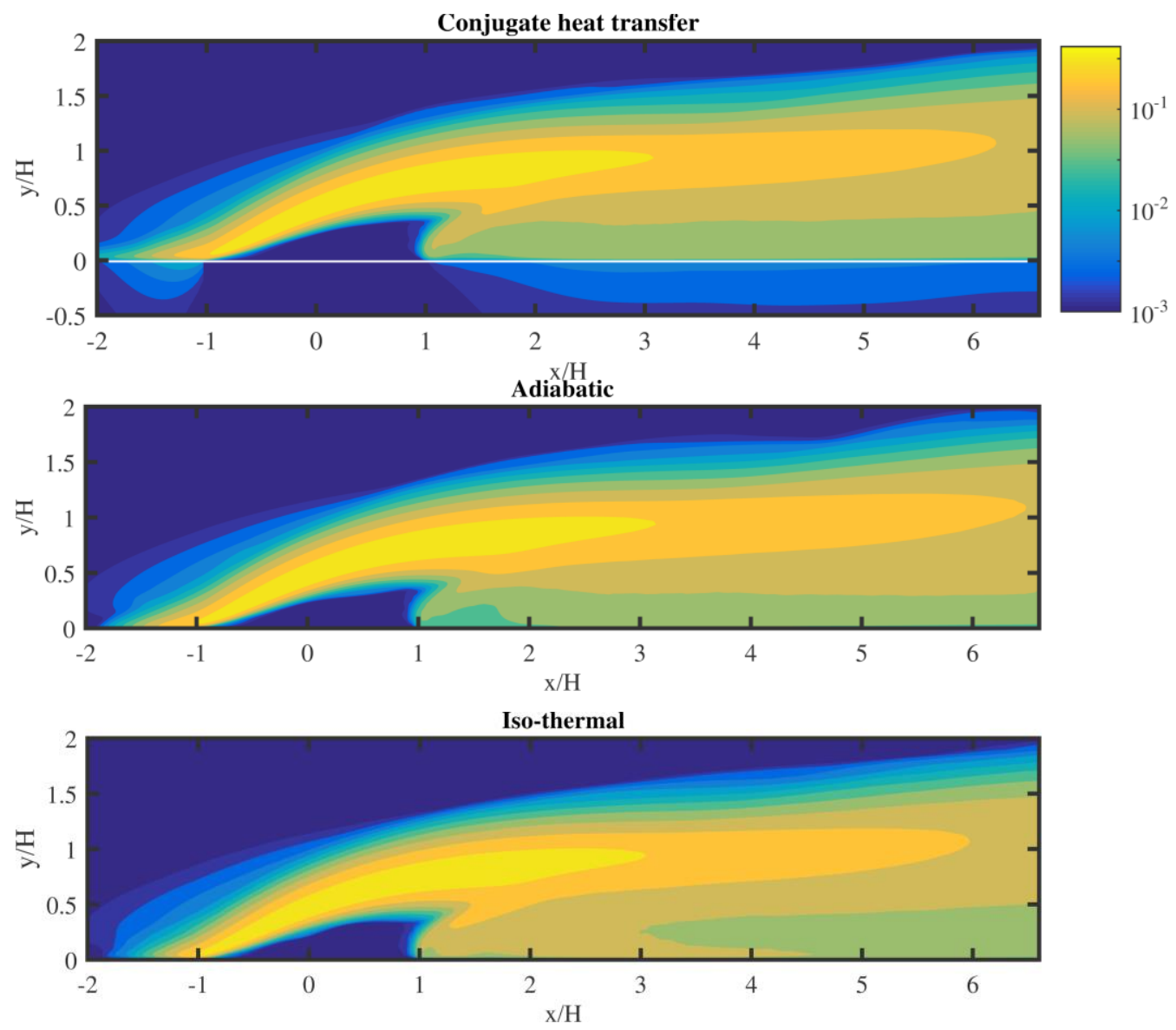

Figure 7. Non-dimensional temperature r.m.s $\theta_{\text {r.m.s }}$ on the symmetry plane in conjugate heat transfer, adiabatic and iso-thermal cases. Plotted in logarithmic scale.

chosen parameters $\left(G, \lambda_{s} / \lambda_{f}\right.$, wall thickness, etc. $)$, and this should not be extended to other conjugate heat transfer cases [47].

12 In most Reynolds-averaged simulations, the time-averaged scalar equation is closed by introducing a turbulent diffusivity $\alpha_{t}=-\left\langle u_{i}^{\prime} \theta^{\prime}\right\rangle / \partial_{i}\langle\theta\rangle$. This value is often simply evaluated as $\alpha_{t}=v_{t} / \operatorname{Pr}_{\mathrm{t}}$ where $\operatorname{Pr}_{\mathrm{t}}>0$ is the turbulent Prandtl number, and thus always positive. However, this is not the case in the current flow: in some regions $\left\langle u_{i}^{\prime} \theta\right\rangle$ and $\partial_{i}\langle\theta\rangle$ have an 
1 angle less than $90^{\circ}$, which would result in negative $\alpha_{t}$ from the above relationship (see Figure

2 11). Simple scalar flux models, therefore, are not expected to work well in these regions.
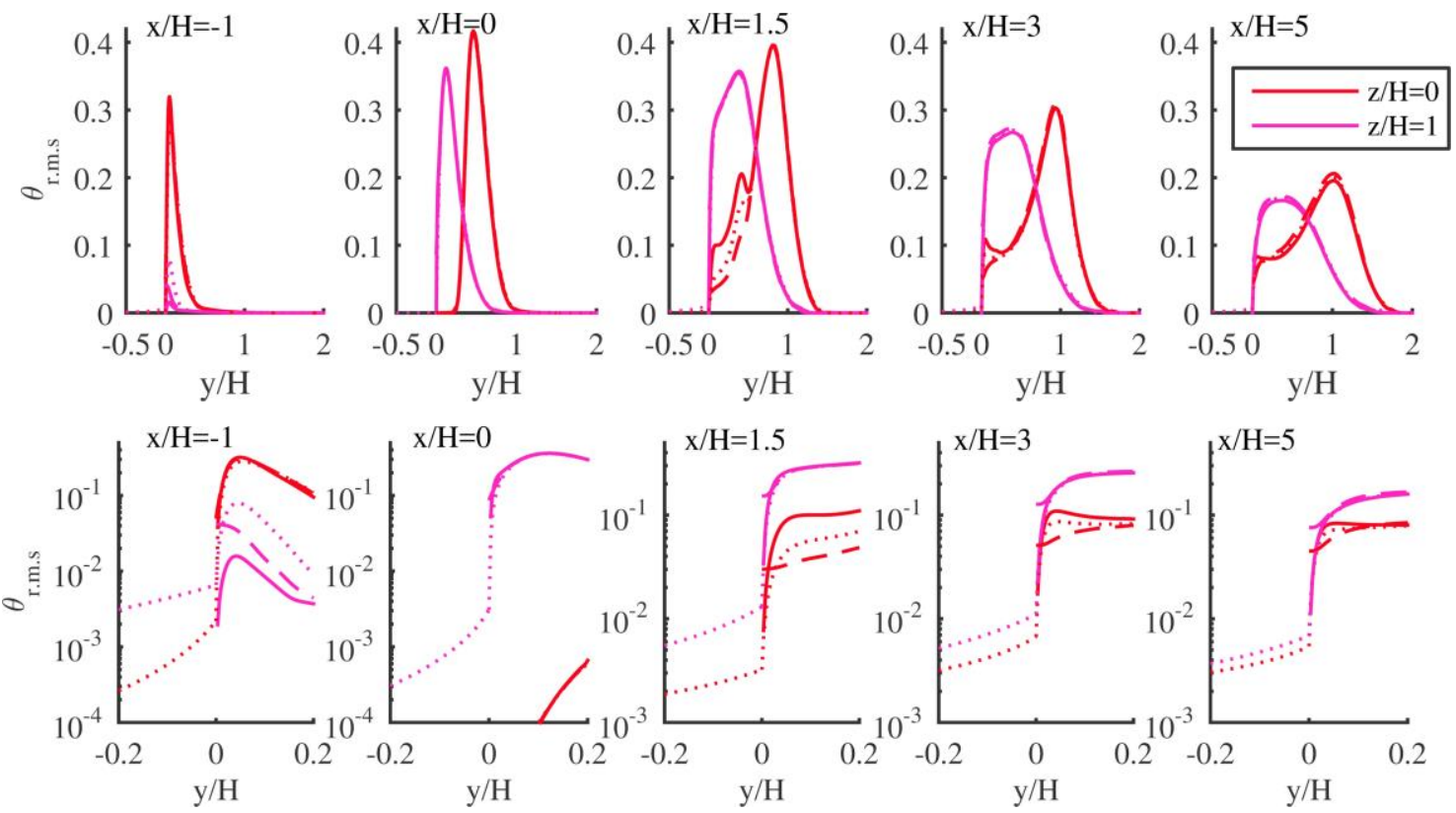

Figure 8. Non-dimensional temperature r.m.s $\theta_{\text {r.m.s }}$ on $z / H=0$ and $z / H=1$. Solid: iso-thermal; dashed: adiabatic; dotted: conjugate heat transfer. Top: linear scale; bottom: zoom in near the interface with log scale for temperatures r.m.s.
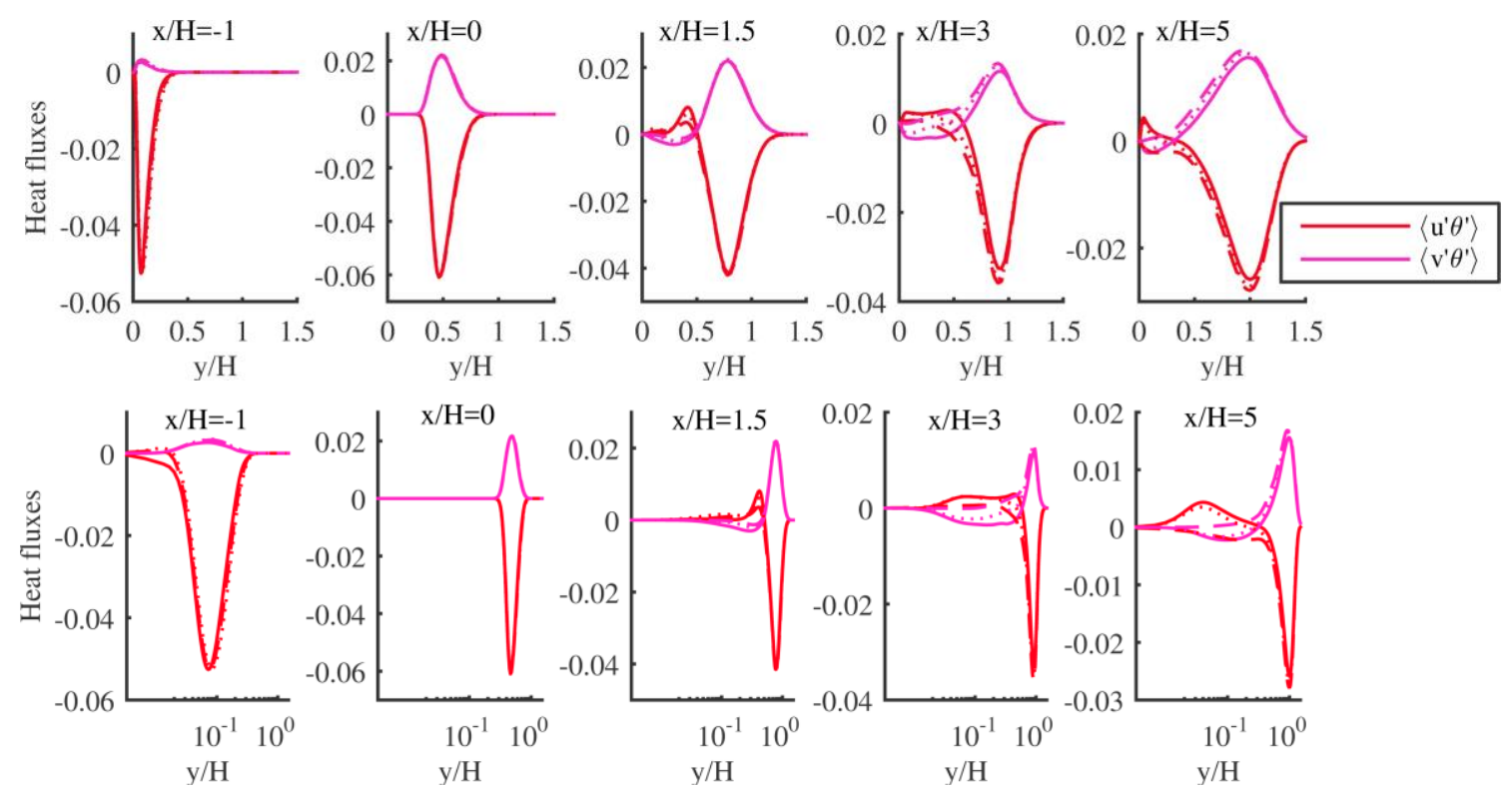

Figure 9. Heat fluxes normalised by $\left\langle u_{c}\right\rangle\left(\theta_{j}-\theta_{c}\right)$ on symmetry plane $(z / H=0)$. Solid: iso-thermal; dashed: adiabatic; dotted: conjugate heat transfer. Top: linear scales; bottom, log scales. $\left\langle u_{c}\right\rangle\left(\theta_{j}-\theta_{c}\right)$. 

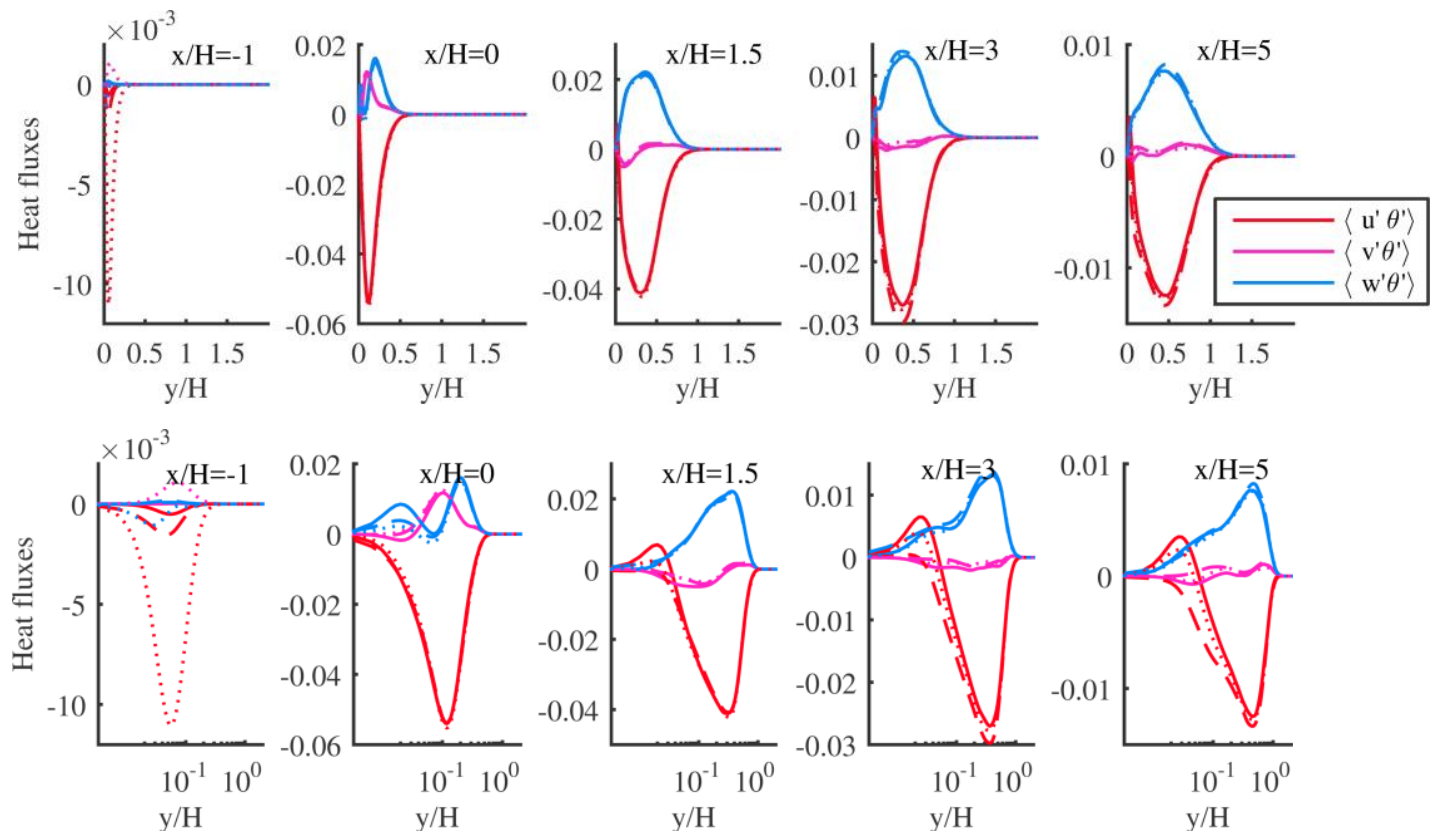

Figure 10. Heat fluxes normalised by $\left\langle u_{c}\right\rangle\left(\theta_{j}-\theta_{c}\right)$ on spanwise plane $(z / H=1)$. Solid: iso-thermal; dashed: adiabatic; dotted: conjugate heat transfer. Top: linear scales; bottom, log scales.

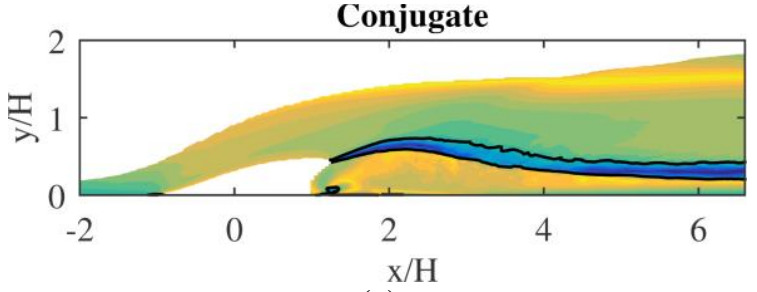

(a)

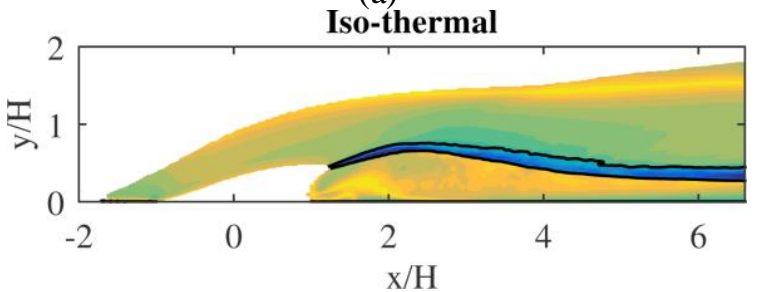

(c)

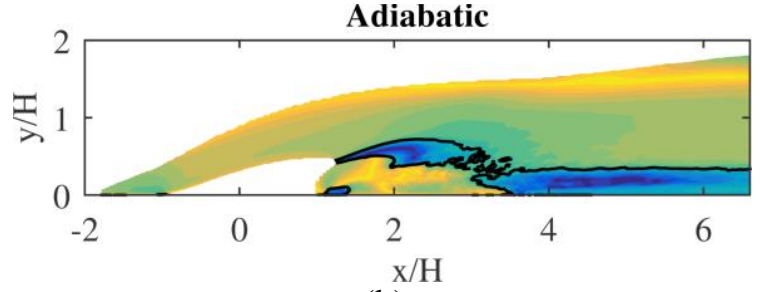

(b)

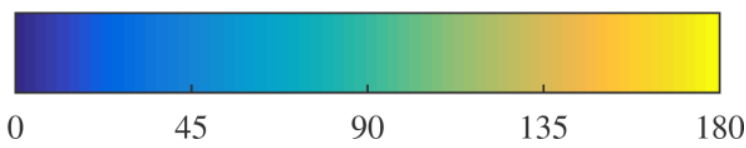

(d)

Figure 11. Angle between heat flux vector $\left\langle u_{i} \theta\right\rangle$ and temperature gradient vector $\partial_{i}\langle\theta\rangle$ on the symmetry plane $(z / H=0)$ in (a) conjugate heat transfer (b) adiabatic and (c) iso-thermal cases with (d) colourmap. The white area is the region with small temperature gradients $\left(\left\|\partial_{i}\langle\theta\rangle\right\|<0.01\left(\theta_{j}-\theta_{c}\right) / H\right)$ where the angle is ill-defined. The black lines in the contour plots are the $90^{\circ}$ contour line between which turbulent diffusivity would be negative.

3 The dissipation of temperature fluctuations, $\varepsilon_{\theta}=\alpha\left\langle\partial_{i} \theta^{\prime} \partial_{i} \theta^{\prime}\right\rangle$, normalized by $\alpha_{f}\left\langle\theta_{j}-\theta_{c}\right\rangle^{2} /$

$4 H^{2}$, is presented in Figure 12 to 13 . In the solid, the transport equation of $\left\langle\theta^{2}\right\rangle$ becomes:

$$
\partial_{t}\left\langle\theta^{\prime 2}\right\rangle=\alpha_{s}\left(\partial_{i i}\left\langle\theta^{\prime 2}\right\rangle-2\left\langle\partial_{i} \theta^{\prime} \partial_{i} \theta^{\prime}\right\rangle\right) .
$$

5 The conjugate heat transfer and iso-thermal cases show similar patterns of wall $\varepsilon_{\theta}$. In the iso-

6 thermal case, a ring appears in the downstream near field because of the recirculation after 
1 the jet (see Figure 12(b)). Figure 13 shows the normalized $\varepsilon_{\theta}$ at several locations. Large 2 dissipation values are seen near the wall as typically found in channel flow (e.g. $[47,48]$ ). The second peak higher above is strongly associated with the shear layer as seen in the temperature r.m.s profiles (Figure 8). Discontinuities of $\varepsilon_{\theta}$ are observed at the interface in the conjugate heat transfer case (bottom of Figure 13). Flageul et al. [32] estimated the ratio $\varepsilon_{\theta, s} / \varepsilon_{\theta, f} \sim \frac{1}{G}\left(\lambda_{f}^{2} / \lambda_{s}^{2}\right)$, where $\varepsilon_{\theta, s}$ and $\varepsilon_{\theta, f}$ represent the dissipation in the solid and fluid sides at the interface respectively. Though the above relation has been derived for a simple channel flow by assuming the wall-parallel part of $\varepsilon_{\theta, f}$ is negligible at the interface, it still appears to apply to present results of complex 3D flow; the value of $\left(\varepsilon_{\theta, s} / \varepsilon_{\theta, f}\right) /\left(\frac{1}{G}\left(\lambda_{f}^{2} / \lambda_{s}^{2}\right)\right)$ is around unity on the fluid-solid interface in the conjugate heat transfer case (Figure 14). The relative contribution of $\varepsilon_{\theta, f}$ 's normal component is also plotted in Figure 15, which shows that the assumption remains valid for the present case.

\subsection{Length and time scales of temperature fluctuations}

The dissipation rate of the temperature variance within the solid layer, $\varepsilon_{\theta, s}=\alpha_{s}\left\langle\partial_{i} \theta^{\prime} \partial_{i} \theta^{\prime}\right\rangle$ needs to be modelled to close the $\left\langle\theta^{\prime 2}\right\rangle$ equation in the solid, as shown for example in Craft et al. [21]. However, it is discontinuous at the interface whereas the interface flux $\lambda_{s} \partial_{n} \theta_{s}^{\prime}=$ $\lambda_{f} \partial_{n} \theta_{f}^{\prime}$ is continuous. The equation of $\theta^{\prime}$ (Eq. (6)) in the solid is linear, in spectral space each wavenumber and frequency mode decays independently from the others (no convolution), so $\varepsilon_{\theta}$ in Eq. (6) only appears as an artefact of transforming $\left\langle\theta^{\prime} \partial_{i i} \theta^{\prime}\right\rangle$ into conduction of $\left\langle\theta^{\prime 2}\right\rangle$ (i.e. $\left\langle\theta^{\prime} \partial_{t} \theta^{\prime}\right\rangle=\alpha_{s}\left\langle\theta^{\prime} \partial_{i i} \theta^{\prime}\right\rangle=\alpha_{s}\left(\frac{1}{2}\left\langle\partial_{i i}\left(\theta^{\prime 2}\right)\right\rangle-\left\langle\partial_{i} \theta^{\prime} \partial_{i} \theta^{\prime}\right\rangle\right)$ ). Craft et al. [21] added an extra sink term to reduce $\varepsilon_{\theta}$, effectively increasing the size of thermal spots and subsequently increasing $\left\langle\theta^{\prime 2}\right\rangle$ penetration. However, as there is no energy cascade in the solid $\varepsilon_{\theta}$ can no longer be related to large-scale fluctuations, i.e. $\varepsilon_{\theta}$ cannot provide an integral length or timescale but the only the dissipative ones. On the other hand, large-scale instantaneous thermal spots should show some continuity across the interface but this is a challenge for RANS one point closures.

Many researchers, e.g. $[49,50]$, considered that $E_{\theta}(\kappa)=C \varepsilon_{\theta} \varepsilon^{-1 / 3} \kappa^{-5 / 3} f(k \eta$, Pr $)$ for $\operatorname{Pr}<1$ perhaps analogous to the low fluid/solid diffusivity ratio of our case. $f(k \eta, \operatorname{Pr})$ may be called the compensated spectrum model, displaying a plateau in the inertial range of fluid turbulence then an exponential decay in the dissipative-diffusion range. 

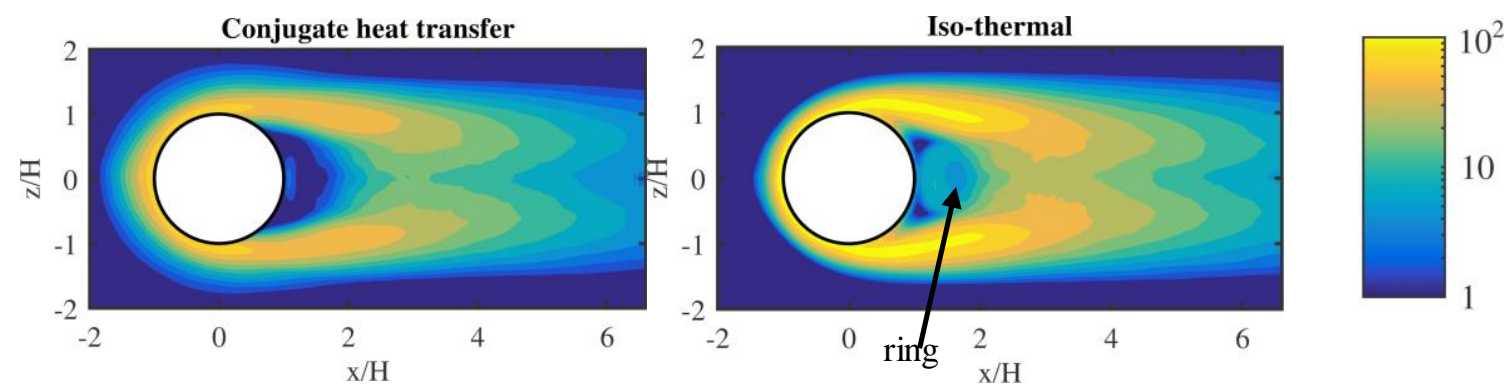

Figure 12. Wall dissipations of temperature fluctuations normalised by $\alpha_{f}\left\langle\theta_{j}-\theta_{c}\right\rangle^{2} / H^{2}$ on the fluid-solid interface in conjugate heat transfer (left) and iso-thermal (right) cases.
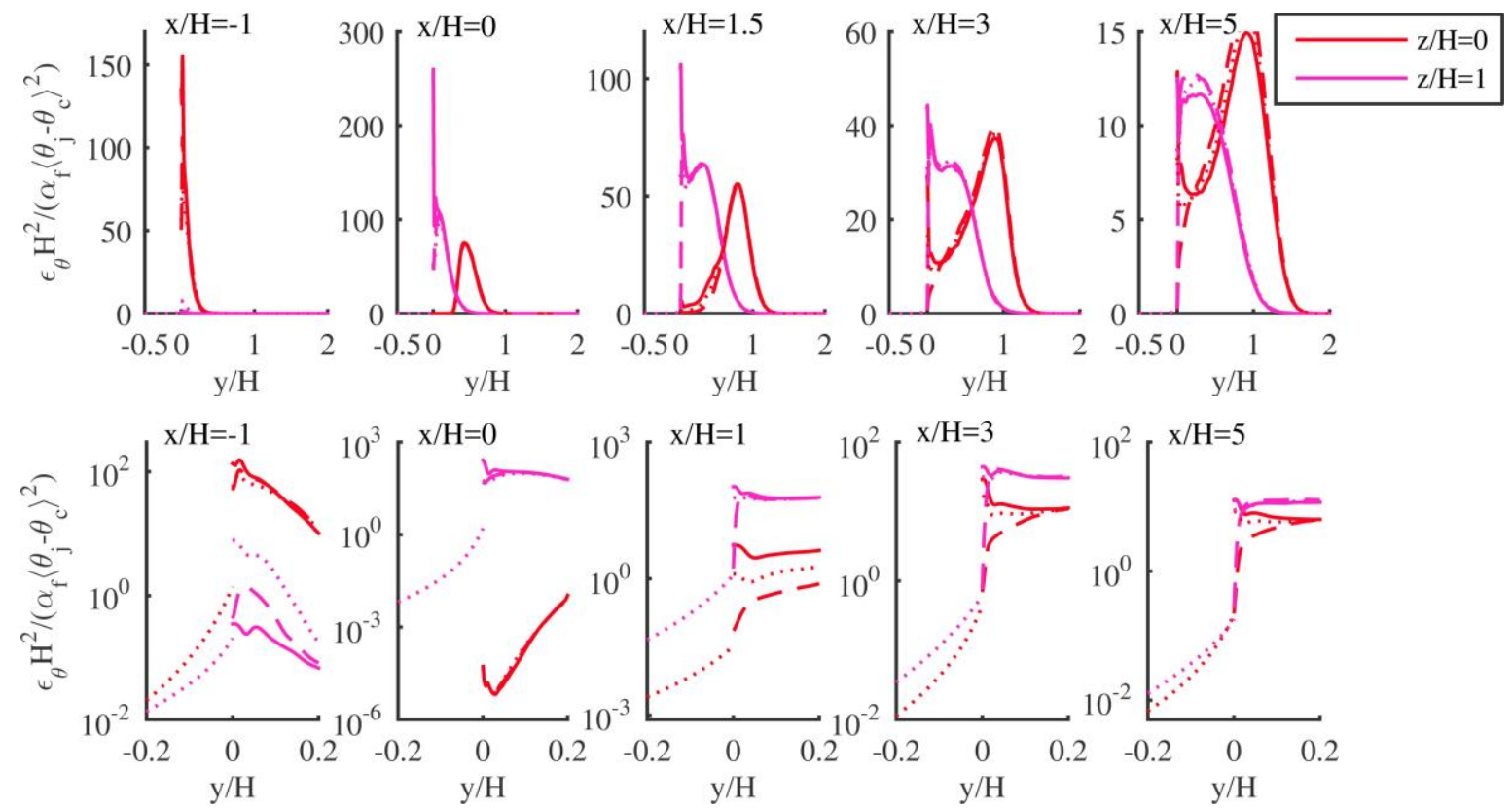

Figure 13. Dissipation of temperature fluctuations $\varepsilon_{\theta}$ normalised by $\alpha_{f}\left\langle\theta_{j}-\theta_{c}\right\rangle^{2} / H^{2}$ on $z / H=0$ and $z / H=1$. Solid: iso-thermal; dashed: adiabatic; dotted: conjugate heat transfer. Top: linear scale; bottom: zoom in near the interface with $\log$ scale for $\varepsilon_{\theta}$.

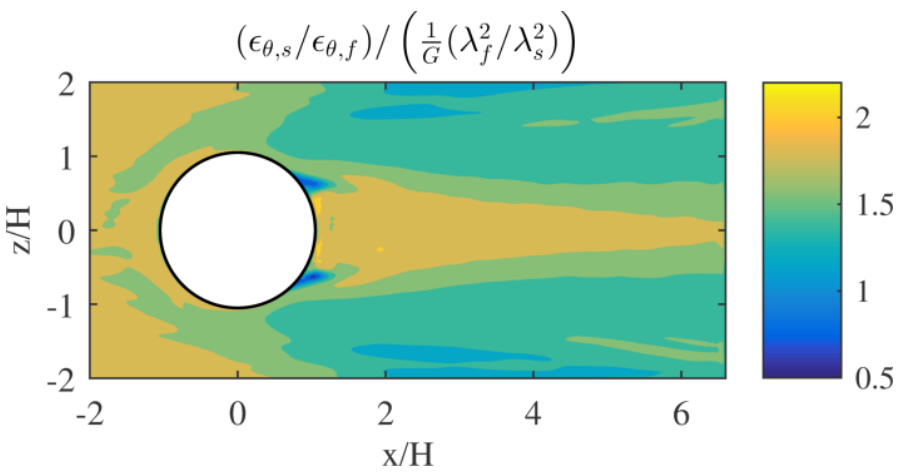

Figure 14. The value of $\left(\varepsilon_{\theta, s} / \varepsilon_{\theta, f}\right) /\left(\frac{1}{G}\left(\lambda_{f}^{2} / \lambda_{s}^{2}\right)\right)$ on the fluid-solid interface in the conjugate heat transfer case. 


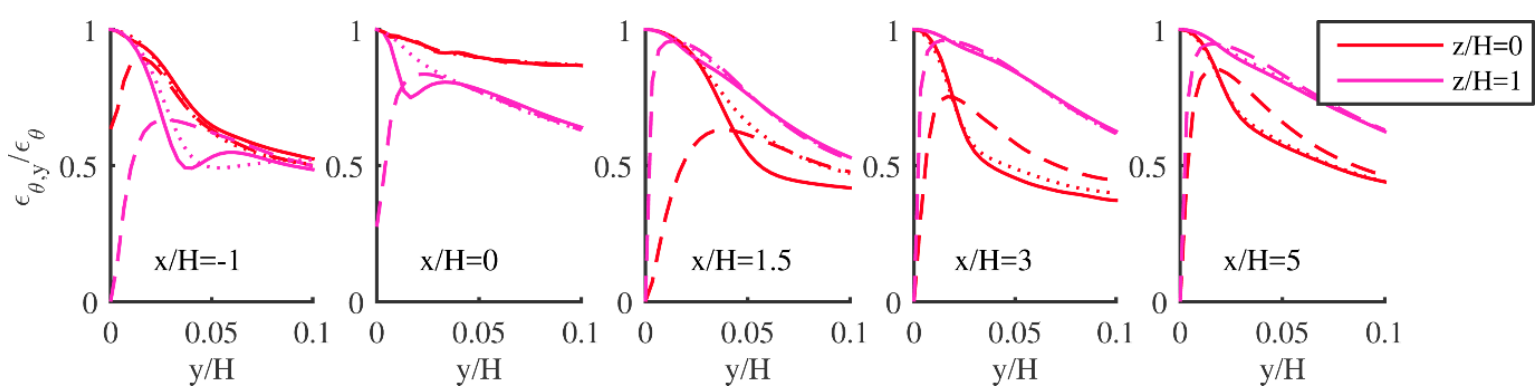

Figure 15. Relative contribution of $\varepsilon_{\theta}$ 's normal component, $\left\langle\partial_{y} \theta^{\prime} \partial_{y} \theta^{\prime}\right\rangle /\left\langle\partial_{i} \theta^{\prime} \partial_{i} \theta^{\prime}\right\rangle$, on $z / H=0$ and $z / H=$ 1. Solid: iso-thermal; dashed: adiabatic; dotted: conjugate heat transfer.
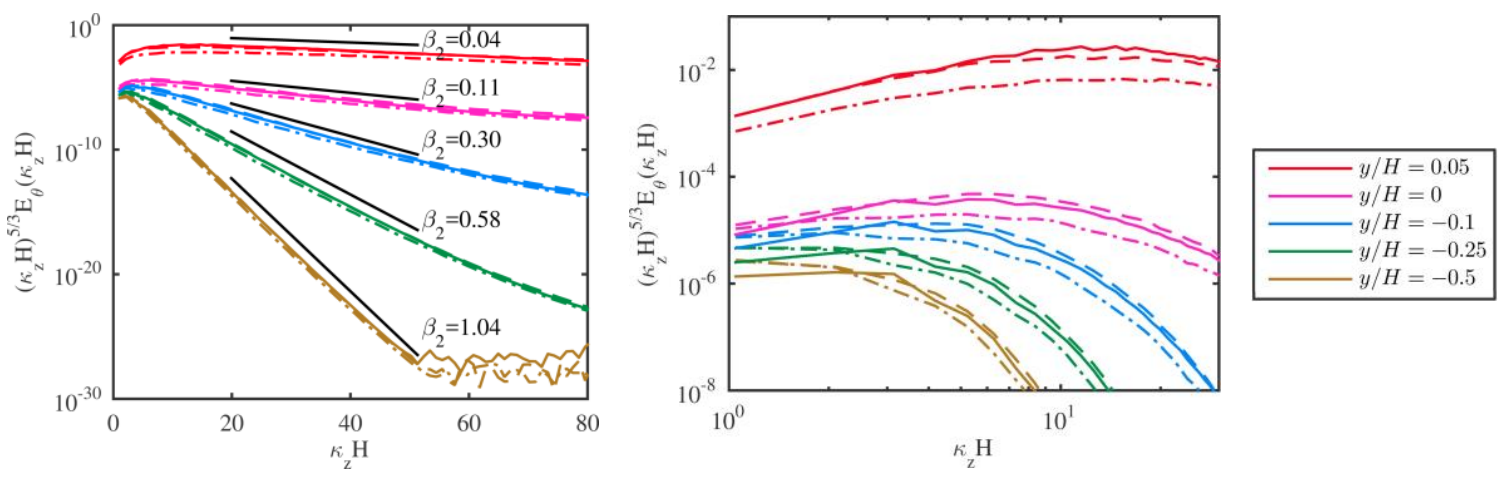

(a)

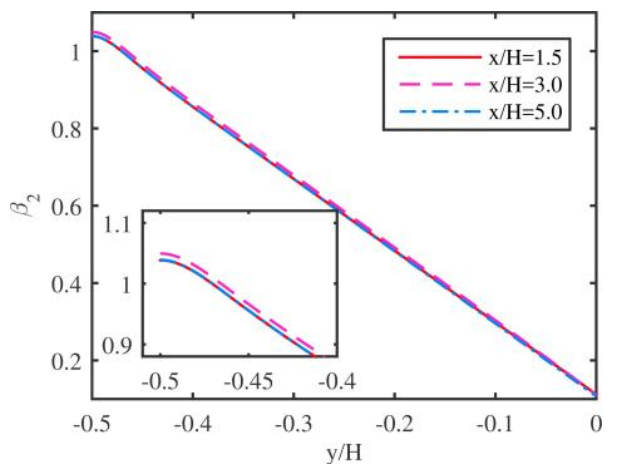

(b)

Figure 16. (a) Compensated one-dimensional temperature spectra in spanwise direction. Solid, $x / H=1.5$; dashed, $x / H=3$; dash-dotted, $x / H=5$. From top to bottom: $y / H=0.05$ (red), $y / H=0$ (purple), $y / H=$ -0.1 (blue), $y / H=-0.25$ (green), $y / H=-0.5$ (yellow); (b) $\beta_{2}$ vs the normal location in solid.

3 Pope suggested that the compensated spectrum, $\kappa^{5 / 3} E(\kappa)$, follows an exponential decay in

4 the dissipation range of velocity spectra, for which he introduced the model (see Section 6.5.3 5 of [51]):

$$
\kappa^{5 / 3} E(\kappa)=f_{\eta}=C_{1} \exp \left(-\beta_{1} \kappa \eta\right) .
$$

6 This exponential decay in the velocity spectra has been checked and validated for the current 7 complex flow in [17]. The temperature spectra are also expected to behave similarly in the 8 Kolmogorov range in both fluid and solid domains due to the dissipation. The compensated 
1 one-dimensional temperature spectra, $\left(\kappa_{z} H\right)^{5 / 3} E_{\theta}\left(\kappa_{z} H\right)$ at locations $(x / H, y / H)$, are 2 presented in Figure 16(a). The spectra are compared at three downstream locations $(x / H=$ $3 \quad 1.5,3$ and 5) and several wall-normal locations from very near the fluid-solid interface in 4 fluid $(y / H=0.05)$ to the outer solid wall $(y / H=-0.5)$. By using a linear-log graph (left of 5 Figure 16(a)), the exponential decay in the diffusive range now becomes obvious. Firstly, the temperature fluctuation versus wavenumber decays faster as one approaches the outer solid wall. Secondly, the temperature fluctuation spectrum at a fixed wall-normal location (same colour in Figure 16(a)) does not vary much as one goes downstream; the spectrum remains of the same order of magnitude at downstream locations. This feature is not established at the centre of channel where $E_{\theta}\left(\kappa_{z}\right)$ far downstream can be one order of magnitude higher than that at near downstream. Thirdly, the exponential decay model, Eq. (7), plotted with a black line, has been rewritten as $f_{\eta}=C_{2} \exp \left(-\beta_{2} \kappa H\right)$, where $C_{2}$ is a constant and $\beta_{2}$ the exponential decay rate exponent. It is found that $\beta_{2}$ at a fixed wall-normal location retains the same value in the downstream direction. The $\beta_{2}$ in solid variation at different $y / H$ locations are presented in Figure 16(b). A linear relationship

$$
\beta_{2}=0.11-1.87 y / H\left(R^{2}=0.9998\right)
$$
is seen in the region $-0.4<y / H<0$, which excludes the outer-wall region where the boundary conditions may largely affect the thermal conditions. Lastly, the oscillations below $10^{-25}$ in Figure 16(a) indicate the accuracy of DNS code is down to machine precision of $10^{-32}$ (the spectrum is pre-multiplied by $\left(\kappa_{z} H\right)^{5 / 3}$ ).

The right graph of Figure 16(a) shows the compensated spectra on a log-log scale with a zoom in the low-wavenumber range. The peak of the compensated spectra corresponds to the wavelength of the energy-containing structures. This plot highlights the large scales in the wall fluid layer, $y / H=0.05$ (red curves), with a maximum at $\kappa_{z} H \sim 10$, i.e. the scale of larger thermal spots is $\sim 0.6 \mathrm{H}$. Penetrating into the fluid this maximum shifts toward even larger scales of the order $H$, quite logically, although this could be an artefact of the 1D $z$ direction spectrum which contains contributions from small wave vectors (large structures) aligned close to the $x$ and $y$ directions and is thus non-zero at the origin [52].

Donzis et al. [49] compiled, for a range of Re and Pr numbers, simulation and experimental data for simple homogeneous isotropic turbulence with constant mean temperature gradient "because the situation with inhomogeneous flows is more complex [but with the] expectation 
1 is that the asymptotic independence discussed here will hold for all flows far from a solid 2 boundary". They defined the scalar integral scale $L_{\theta_{-} 2 p t}=\frac{\pi}{2\left\langle\theta^{\prime 2}\right\rangle} \int \frac{E_{\theta}(\kappa)}{\kappa} \mathrm{d} \kappa$, or simply 3 microscale $L_{\theta_{-}} 1 p t=\frac{\left\langle\theta^{\prime 2}\right\rangle^{1 / 2}}{\sqrt{\left\langle\partial_{i} \theta\right\rangle^{2}}}$ for data where the spherical integrated $3 \mathrm{D}$ spectrum is not 4 available (they defined the latter one as another integral scale, although it is actually a Taylor 5 microscale [8] but is accessible to one-point closure modelling).

6 Our jet in channel crossflow is very far from the above homogeneous case and 3D spectra are 7 meaningless. Length scales could be obtained by two-point correlations but these require 8 huge sample sizes, as only time averaging is possible here. The similarity of the above scales 9 is qualitatively tested using the $z$-direction spectrum previously shown in Figure 16 (a). That 10 is, the two-point length scale is defined as

$$
L_{\theta \_2 p t}=\frac{\pi}{2 \frac{1}{L_{z}} \int\left\langle\theta^{\prime 2}\right\rangle \mathrm{d} z} \int \frac{1}{\kappa_{z}} E_{\theta}\left(\kappa_{z}\right) \mathrm{d} \kappa_{z}
$$

11 since $1 \mathrm{D}$ to $3 \mathrm{D}$ transform relations require derivatives of the $1 \mathrm{D}$ spectrum which is not 12 smooth enough for this purpose and in any case the $1 \mathrm{D}-3 \mathrm{D}$ relation is only valid for the 13 homogeneous isotropic case. The one-point closure scale is defined as

$$
L_{\theta_{-} 1 p t}=\frac{\left(\frac{1}{L_{z}} \int\left\langle\theta^{\prime 2}\right\rangle \mathrm{d} z\right)^{1 / 2}}{\frac{1}{L_{z}} \int\left(\partial_{n}\langle\theta\rangle \partial_{n}\langle\theta\rangle\right)^{1 / 2} \mathrm{~d} z}
$$
analogous to Donzis et al. [49]'s definition. Here, this length scale is a model which is defined using the quantities available in the RANS models. Both length scales, normalised by $H$, are shown in Figure 17.

17 On the fluid-solid interface $(y / H=0)$, discontinuities are seen in the $L_{\theta_{-} 1 p t}$ profiles. The modelled length scale $L_{\theta_{-} 1 p t}$ is discontinuous on the interface due to the discontinuity of the temperature gradient. The length scales of hot spots predicted by $L_{\theta \_} 2 p t$ are closer to that determined from the compensated spectra near $y / H=0$. Both $L_{\theta_{-} 2 p t}$ and $L_{\theta_{-} 1 p t}$ length scales almost conserve their magnitudes as one goes deeper in the solid wall. However, the one-point relations underestimated the length scales in solid by a factor of more than two. 


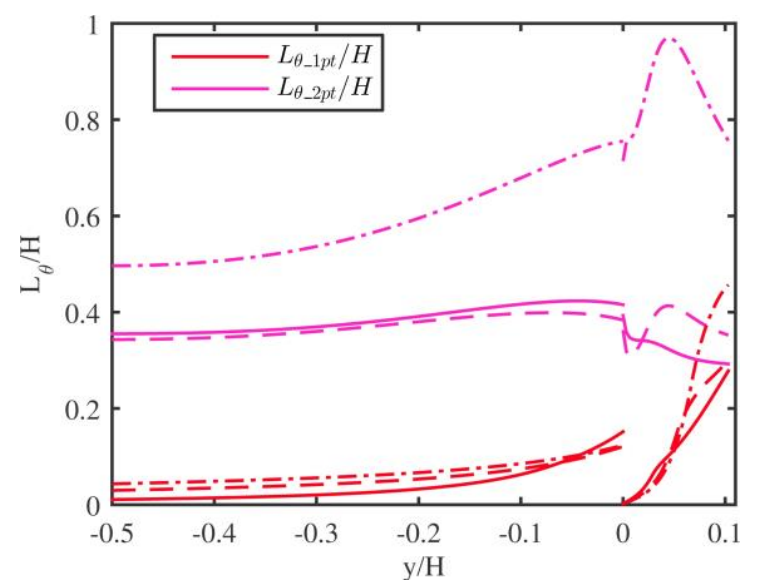

Figure 17. Length scale normalised by $H$ calculated by Eq. (7) and (8). Solid, $x / H=1.5$; dashed, $x / H=3$; dash-dotted, $x / H=5$.

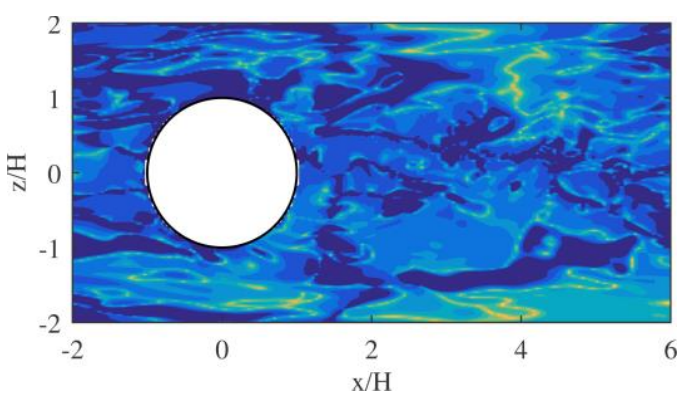

(a)

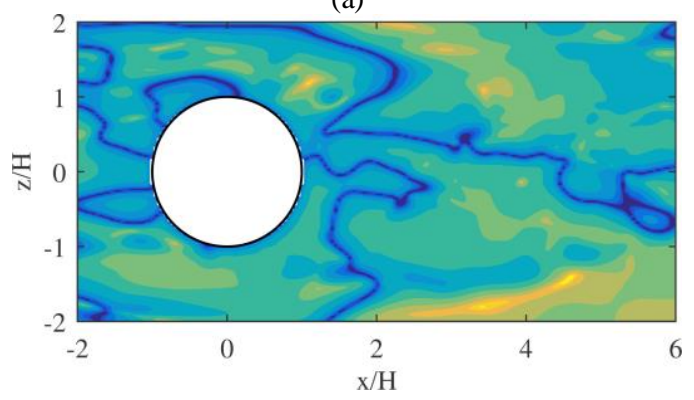

(c)

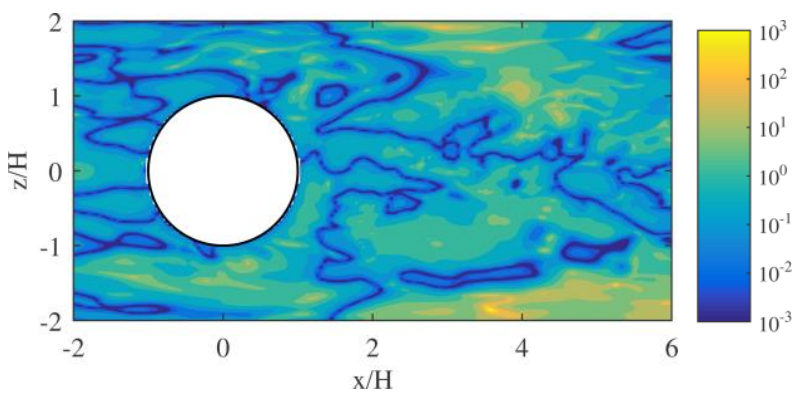

(b)

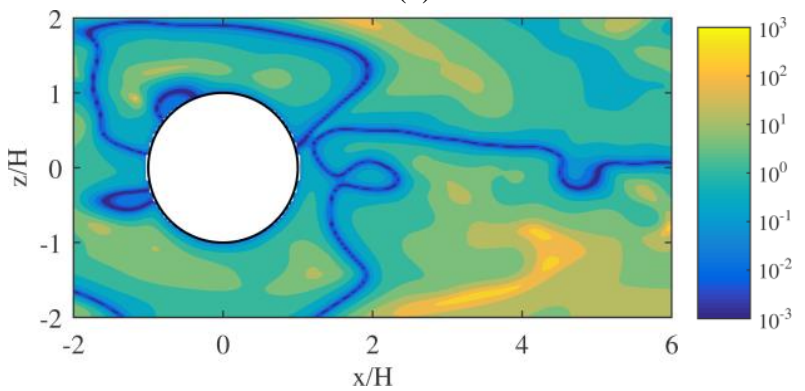

(d)

Figure 18. Instantaneous timescale $\theta^{\prime 2} / \varepsilon_{\theta}^{\prime}$, normalized by $H /\left\langle u_{c}\right\rangle$, at $t=t_{0}$ on (a) $y / H=0$ (calculated by fluid $\theta^{\prime}$ and $\varepsilon_{\theta}^{\prime}$ ), (b) $y / H=0$ (calculated by solid $\theta^{\prime}$ and $\varepsilon_{\theta}^{\prime}$ ), (c) $y / H=-0.1$ and (d) $y / H=-0.25$.

3 The quantity $\left\langle\theta^{\prime 2}\right\rangle / \varepsilon_{\theta}$ is normally used to produce a mechanical-to-thermal time scale ratio $4(k / \varepsilon) /\left(\left\langle\theta^{\prime 2}\right\rangle / \varepsilon_{\theta}\right)$ (e.g. $\left.[49,53-55]\right)$. All these studies use the time-averaged value. Here we 5 use the instantaneous value to study the temperature field in solid. The instantaneous time scale $\theta^{\prime 2} / \varepsilon_{\theta}^{\prime}$, normalized by $H /\left\langle u_{c}\right\rangle$, is shown in Figure $18(\mathrm{a}-\mathrm{d})$ with the logarithmic colormap at several $y / H$ locations. On the fluid-solid interface $(y / H=0)$, the time scales are calculated for both the fluid and solid values. Blue to yellow contours represent small to large time scales. Discontinuity of time scales is clearly seen on $y / H=0$ across the fluid-solid interface, obviously due to the jump of dissipation rate. Intense dissipation-diffusion 
1 corresponds to short time scales (blue colour). Magnitude of $\theta^{\prime 2} / \varepsilon_{\theta}^{\prime}$ in the solid can no 2 longer be related to large-scale structures as there is no longer an energy cascade to link 3 dissipation to large scales but its topology is relevant to borders of large-scale thermal structures (perhaps analogous to non-premixed reacting flows where this time scale is widely used). Flageul et al. [28] concluded only large-scale structures with long lifetime are able to penetrate deeply inside the solid wall by analysing the temperature autocorrelations on their channel flow. This conclusion is supported in this study by the instantaneous profiles: it is clearly observed that the long lifetime structures are "evenly" distributed in the fluid, but more localised in the solid (Figure 18(b-d)). In fact, since the heat diffusion equation is linear, the structures observed in the solid should also be identifiable in the fluid or at least on the fluid/solid interface: indeed, the footprints of time scale structures are seen on the interface (Figure 18(a)). As one goes deeper into the solid, the large structures remain in accordance with the compensated spectra observations in Figure 16(a) and the quasi-flat $L_{\theta}$ profiles in Figure 17. The apparent smoothness of the later may be an artefact long term averaging hiding a very localised and intermittent activity which makes attempts to extend RANS modelling to the solid layer very challenging and perhaps analogous to difficulties of modelling the intermittent upper edge of boundary layers (both problems being dominated by rare large scale structures). Indeed very few structures are seen in the solid part of Figure 18 and one may wonder if the long-time averaging then modelling of $\theta^{2}$ is meaningful, hence we next try to track structures over time.

Figure 19 shows the instantaneous temperature snapshots on three horizontal planes. The "thermal spots" sizes observed qualitatively correspond to the large structures' length scales, $L_{\theta \_2 p t}$, defined by integrating the spanwise spectra. The top row of Figure 19 shows the temperature fluctuation maps on a horizontal plane in fluid $y / H=0.05$ (or $y^{+} \sim 10$ ). Thermal spots are seen generated around the jet-exit where the crossflow encounters the jet fluid.

In the fluid, three fluid fluctuation structures are circled by the dashed ellipses (top row of Figure 19). In the solid (middle and bottom rows of Figure 19), these structures are also traced, illustrating the correlation between the fluid and solid domains. As it is well known, the low-frequency temperature fluctuations penetrate deeper through the solid wall, see for example Lee et al. [44] for T-junctions. Similarly, only large structures remain in the solid for the presently chosen values of $\operatorname{Re}, G$, conductivity ratio and wall thickness. It is also noticed that the thermal structures in the solid are almost always somewhat upstream over the 
1 corresponding spots in the fluid. This illustrates a time-delay effect in the fluid-solid 2 correlation.

3 On the fluid-solid interface (middle row of Figure 19), A1 points out a thermal spot. As the 4 flow evolves (from the left column to right), the A1 spot moves to downstream (A2 and A3); 5 this structure seems to be carried downstream by the "fluid". Since there is no convection in 6 the solid, these spots are mirroring those transported in the fluid domain via fluid-solid 7 couplings. The convective effect is also seen in the solid, e.g. B1, B2 and B3 on $y / H=$ $8-0.15$ (bottom row of Figure 19).

9 The instantaneous near-wall $(y / H<0.1)$ iso-surface of $Q$-criterion and the wall Nusselt 10 number are presented in Figure 20 for both the conjugate heat transfer and iso-thermal cases. 11 In the downstream nearfield $(0<x / H<2)$, as the temperature of turbulent structures is seen changing rapidly (black to white in Figure 20) over a short spanwise distance, the temperature gradient is large on the sides of the wake, where the $\mathrm{Nu}$ are best correlated with turbulent structures. The cold crossflow is mixing with the hot jet, and thus higher temperature structures (white coloured iso-Q surfaces) heat the bottom wall. Further downstream $(x / H>2)$, the structures gather near the symmetry plane as the fluid flows inward towards the central plane. Upstream of the jet exit high heat transfer is not induced by turbulence heat diffusion, but by mainly mean flow convective heat transfer effect.

\section{Conclusions}

A conjugate heat transfer DNS code has been developed on the basis of Flageul et al. [28]'s bi-periodic code for conjugate heat transfer in channel flows. The fluid-solid thermal coupling method proposed by [30] has been found simple and efficient.

The main contribution of this paper is that it has revealed the following features of turbulent conjugate heat transfer for a case with strong industrial relevance.

Wall maps of Nusselt number and r.m.s wall-temperature, $\theta_{\text {r.m.s }}$, for conjugate heat transfer are qualitatively similar to those of iso-thermal and adiabatic wall cases, but in the conducting wall case the solid is pre-heated by the hot injection pipe, leading to negative $\mathrm{Nu}$ number upstream of the injection.

Instantaneous temperature fluctuation maps in planes below the interface show some correlation with those in the fluid-flow buffer layer, with some convective and time-delay effects. Thermal spots are generated around the jet-exit where the cold crossflow encounters 
1 the hot jet fluid. The larger and intense thermal spots penetrate all the way across to the outer 2 solid wall while high-frequency fluctuations are smoothed out almost instantly, which is

3 reflected by the discontinuity in temperature variance dissipation across the interface.

4 The magnitude of fluctuating temperature variance spectrum above and below the interface is 5 found to decay exponentially in the diffusion-dissipation range as in the theoretical models 6 for homogeneous fluid turbulence. The exponential decay rates at a fixed wall-normal 7 location are found to remain constant in the downstream direction. This finding implies an empirical function between the exponential decay rate and the wall depth mainly.

9 The large structures' length scales defined by integrating the spanwise spectra seem to 10 correspond to the "hot spots" size seen in the instantaneous temperature snapshots. This two11 point length scale shows very small discontinuities across the interface and could advantageously replace dissipation-based scaling "a la Kolmogorov inertial range" often presumed in RANS models since the later leads to a much larger discontinuity and underestimation of the two-point length scale.

15 A higher Re JICF DNS is needed to confirm this conjecture. Recent high number channel 16 flow simulations show that the viscous and buffer layers are slaves to the larger outer-flow 17 structures, which also strongly contribute to r.m.s wall pressure fluctuations and would logically have a similar impact on the wall temperature pattern.

19 A simpler 2D slot jet, rather than round jet test case, would also facilitate interpretation of 20 spanwise spectra.

\section{Acknowledgements}

22 The authors would like to acknowledge the assistance given by IT Services and the use of the 23 Computational Shared Facility at The University of Manchester. The authors acknowledge the use of ARCHER HPC allocated via UK EPSRC Turbulence Consortium (EPSRC grant EP/L000261/1) and the use of BlueGene/Q supercomputer sponsored by EDF R\&D centre Chatou. The authors are grateful to Cedric Flageul for his invaluable help on this project. 

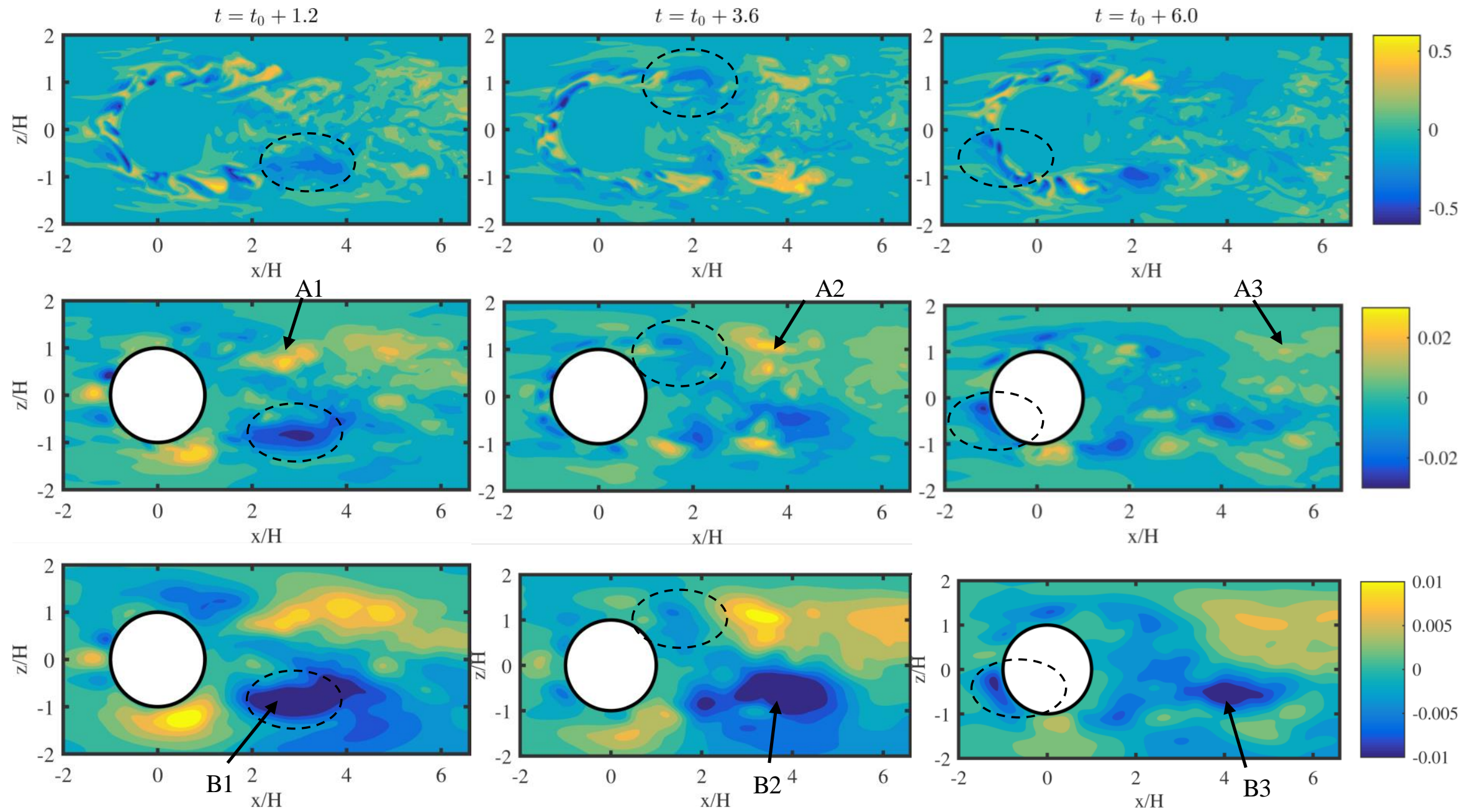

Figure 19. Instantaneous temperature fluctuations in conjugate heat transfer case at three time-steps. Top: in the fluid domain near the wall $(y / H=0.05)$; middle: on the fluid-solid interface $(y / H=0)$; bottom: on the solid outer wall $(y / H=-0.15)$. 

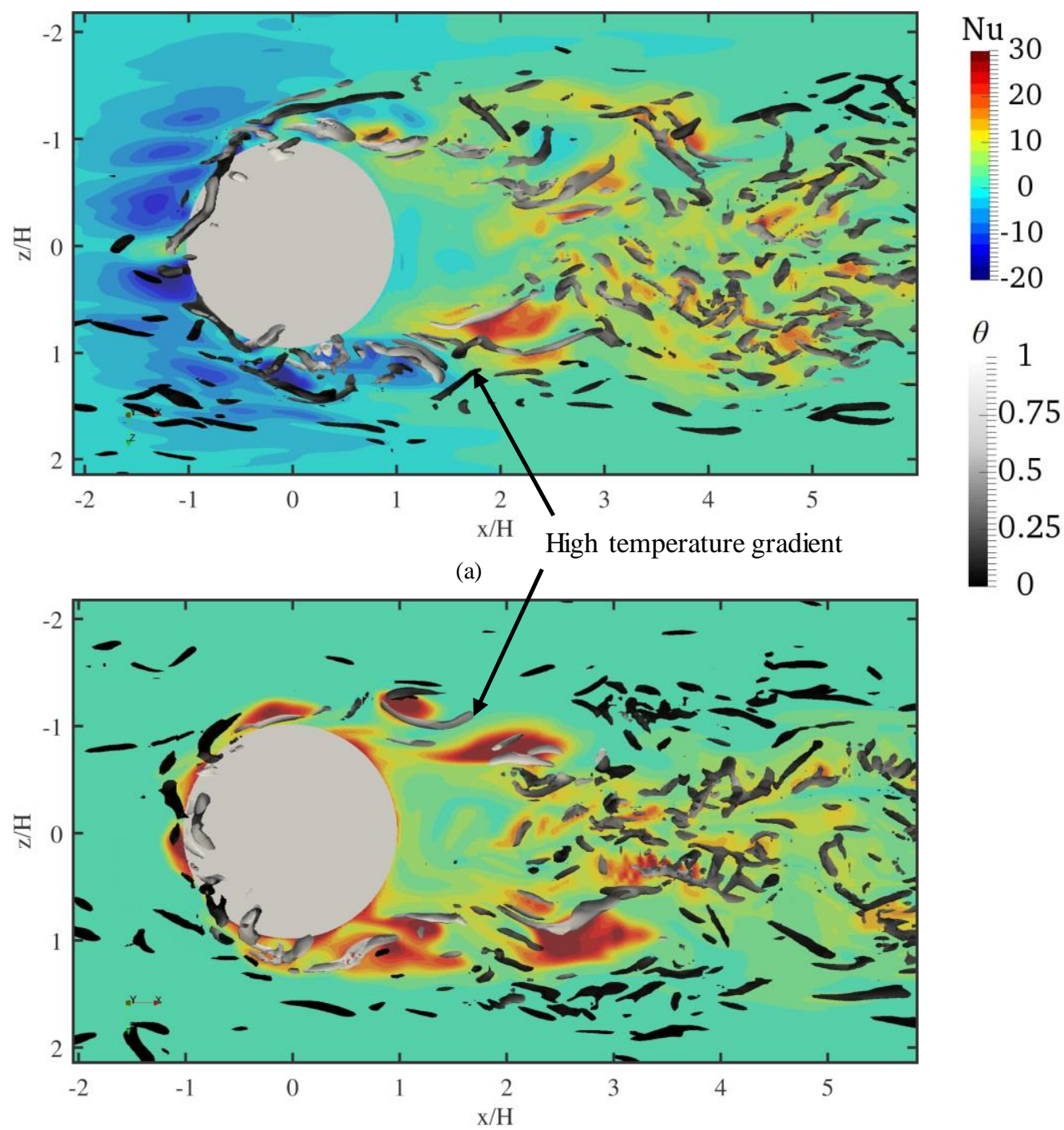

(b)

Figure 20. Instantaneous iso-surfaces of $Q$-criterion $(Q=5)$ and wall Nusselt number at $t=t_{0}$ in (a) conjugate heat transfer case and (b) iso-thermal case. Only the near-wall iso- $Q(y / H<0.1)$, coloured by the dimensionless temperature $\theta$ (black to white: low to high $\theta$ ), is presented.

\section{References}

3 [1] S.A. Sherif, R.H. Pletcher, Measurements of the Flow and Turbulence Characteristics of Round Jets in Crossflow, J. Fluids Eng. 111 (1989) 165-171. doi:10.1115/1.3243618.

[2] T.F. Fric, A. Roshko, Vortical structure in the wake of a transverse jet, J. Fluid Mech. 279 (1994) 1-47. doi:10.1017/S0022112094003800.

[3] R.M. Kelso, A.J. Smits, Horseshoe vortex systems resulting from the interaction between a laminar boundary layer and a transverse jet, Phys. Fluids. 7 (1995) 153-158. doi:10.1063/1.868736. 
1 [4] R.M. Kelso, T.T. Lim, A.E. Perry, An experimental study of round jets in cross-flow, J. Fluid Mech. 306 (1996) 111. doi:10.1017/S0022112096001255.

[5] B.A. Haven, M. Kurosaka, Kidney and anti-kidney vortices in crossflow jets, J. Fluid Mech. 352 (1997) 27-64. doi:10.1017/S0022112097007271.

[6] S.H. Smith, M.G. Mungal, Mixing, structure and scaling of the jet in crossflow, J. Fluid Mech. 357 (1998) 83-122. doi:10.1017/S0022112097007891.

[8] J.W. Shan, P.E. Dimotakis, Reynolds-number effects and anisotropy in transverse-jet

[9] H. Iacovides, D. Kounadis, B.E. Launder, J. Li, Z. Xu, Experimental Study of the Flow

[10] S. Muppidi, K. Mahesh, Direct numerical simulation of round turbulent jets in

[11] R. Sau, K. Mahesh, Dynamics and mixing of vortex rings in crossflow, J. Fluid Mech. 604 (2008) 389-409. doi:10.1017/S0022112008001328.

[12] M. Esmaeili, A. Afshari, F.A. Jaberi, Turbulent mixing in non-isothermal jet in

[13] G. He, Y. Guo, A.T. Hsu, The effect of Schmidt number on turbulent scalar mixing in a jet-in-crossflow, Int. J. Heat Mass Transf. 42 (1999) 3727-3738. doi:10.1016/S00179310(99)00050-2.

[14] T.J. Craft, H. Iacovides, N.A. Mostafa, Modelling of three-dimensional jet array 26 impingement and heat transfer on a concave surface, Int. J. Heat Fluid Flow. 29 (2008) 687-702. doi:10.1016/j.ijheatfluidflow.2008.03.005.

[15] J. Andreopoulos, W. Rodi, Experimental investigation of jets in a crossflow, J. Fluid Mech. 138 (1984) 93-127. doi:10.1017/S0022112084000057. 
1 [16] B.M. Duda, Étude et analyse numérique d'un jet chaud débouchant dans un écoulement transverse en utilisant des simulations aux échelles résolues, Toulouse, ISAE, 2012.

[17] Z. Wu, D. Laurence, I. Afgan, Direct numerical simulation of a low momentum round jet in channel crossflow, Nucl. Eng. Des. 313 (2017) 273-284. doi:10.1016/j.nucengdes.2016.12.018.

[18] S. Chapuliot, C. Gourdin, T. Payen, J.P. Magnaud, A. Monavon, Hydro-thermalmechanical analysis of thermal fatigue in a mixing tee, Nucl. Eng. Des. 235 (2005) 575-596. doi:10.1016/j.nucengdes.2004.09.011.

[19] J.M. Stephan, F. Curtit, C. Vindeirinho, S. Taheri, M. Akamatsu, C. Peniguel, Evaluation of the Risk of Damages in Mixing Zones: EDF R\&D Programme, in: Fatigue, Fract. Damage Anal. Vol. 1, ASME, 2002: pp. 33-38. doi:10.1115/PVP20021328.

[20] J.-A. Le Duff, A. Lefrançois, Y. Meyzaud, J.-P. Vernot, D. Martin, J. Mendez, Y. Lehericy, High cycle thermal fatigue Issues in PWR nuclear power plants, life time improvement of some austenitic stainless steel components, Rev. Métallurgie. 104 (2007) 156-162. doi:10.1051/metal:2007142.

[21] T.J. Craft, H. Iacovides, S. Uapipatanakul, Towards the development of RANS models for conjugate heat transfer, J. Turbul. $11 \quad$ (2010) N26. doi:10.1080/14685248.2010.494608.

[22] Z. Wu, D. Laurence, I. Afgan, DNS of a jet in cross flow with passive scalar mixing, in: 8th Int. Symp. Turbul. Heat Mass Transf., Begellhouse, Sarajevo, Bosnia and Herzegovina, 2015: pp. 479-482.

[23] Z. Wu, D. Laurence, S. Utyuzhnikov, I. Afgan, Proper orthogo nal decomposition and dynamic mode decomposition of jet in channel crossflow (submitted), J. Fluid Mech. (2017).

[24] Z. Wu, D. Laurence, I. Afgan, Evaluation of RANS Models for Jet in Crossflow (submitted), Int. J. Heat Fluid Flow. (2017).

[25] Z. Wu, D. Laurence, I. Afgan, Direct simulation of hot jet in cold channel crossflow with temperature mixing, Mendeley Data, v2. (2017). doi:10.17632/7nx4prgizz.2. 
1 [26] S. Laizet, E. Lamballais, High-order compact schemes for incompressible flows: A simple and efficient method with quasi-spectral accuracy, J. Comput. Phys. 228 (2009) 5989-6015. doi:10.1016/j.jcp.2009.05.010.

[27] S. Laizet, N. Li, Incompact3d: A powerful tool to tackle turbulence problems with up to $\mathrm{O}(105)$ computational cores, Int. J. Numer. Methods Fluids. 67 (2011) 1735-1757. doi:10.1002/fld.2480.

[28] C. Flageul, S. Benhamadouche, É. Lamballais, D. Laurence, DNS of turbulent channel flow with conjugate heat transfer: Effect of thermal boundary conditions on the second moments and budgets, Int. J. Heat Fluid Flow. 55 (2015) 34-44. doi:10.1016/j.ijheatfluidflow.2015.07.009.

[29] S.K. Lele, Compact finite difference schemes with spectral-like resolution, J. Comput. Phys. 103 (1992) 16-42. doi:10.1016/0021-9991(92)90324-R.

[30] M.B. Giles, Stability analysis of numerical interface conditions in fluid-structure thermal analysis, Int. J. Numer. Methods Fluids. 25 (1997) 421-436. doi:10.1002/(SICI)1097-0363(19970830)25:4<421::AID-FLD557>3.0.CO;2-J.

[31] B. Roe, R. Jaiman, A. Haselbacher, P.H. Geubelle, Combined interface boundary condition method for coupled thermal simulations, Int. J. Numer. Methods Fluids. 57 (2008) 329-354. doi:10.1002/fld.1637.

[32] C. Flageul, Création de bases de données fines par simulation directe pour les effets de la turbulence sur les transferts thermiques pariétaux, Université de Poitiers, 2015.

[33] C. Flageul, S. Benhamadouche, É. Lamballais, D. Laurence, DNS of channel flow with conjugate heat transfer - Budgets of turbulent heat fluxes and temperature variance, in: 10th Int. ERCOFTAC Symp. Eng. Turbul. Model. Meas., Marbella, Spain, 2014.

[34] E. Lamballais, Direct numerical simulation of a turbulent flow in a rotating channel with a sudden expansion, J. Fluid Mech. 745 (2014) 92-131. doi:10.1017/jfm.2014.30.

[35] M. Salewski, D. Stankovic, L. Fuchs, Mixing in circular and non-circular jets in crossflow, Flow, Turbul. Combust. 80 (2008) 255-283. doi:10.1007/s10494-007-9119$\mathrm{X}$.

[36] S. Bagheri, P. Schlatter, P.J. Schmid, D.S. Henningson, Global stability of a jet in crossflow, J. Fluid Mech. 624 (2009) 33. doi:10.1017/S0022112009006053. 
1 [37] F. Muldoon, S. Acharya, Direct Numerical Simulation of pulsed jets-in-crossflow, Comput. Fluids. 39 (2010) 1745-1773. doi:10.1016/j.compfluid.2010.04.008.

[38] R.W. Grout, A. Gruber, C.S. Yoo, J.H. Chen, Direct numerical simulation of flame stabilization downstream of a transverse fuel jet in cross-flow, Proc. Combust. Inst. 33 (2011) 1629-1637. doi:10.1016/j.proci.2010.06.013.

[39] M. Ilak, P. Schlatter, S. Bagheri, D.S. Henningson, Bifurcation and stability analysis of a jet in cross-flow: onset of global instability at a low velocity ratio, J. Fluid Mech. 696 (2012) 94-121. doi:10.1017/jfm.2012.10.

[40] M.A. Ol'shanskii, V.M. Staroverov, On simulation of outflow boundary conditions in finite difference calculations for incompressible fluid, Int. J. Numer. Methods Fluids. 33 (2000) 499-534. doi:10.1002/1097-0363(20000630)33:4<499::AIDFLD19>3.0.CO;2-7.

[41] T. Dairay, V. Fortuné, E. Lamballais, L.-E. Brizzi, Direct numerical simulation of a turbulent jet impinging on a heated wall, J. Fluid Mech. 764 (2015) 362-394. doi:10.1017/jfm.2014.715.

[42] E. Lamballais, J. Silvestrini, S. Laizet, Direct numerical simulation of flow separation behind a rounded leading edge: Study of curvature effects, Int. J. Heat Fluid Flow. 31 (2010) 295-306. doi:10.1016/j.ijheatfluidflow.2009.12.007.

[43] L.C. Pinto, E.B.C. Schettini, J.H. Silvestrini, Numerical analysis of the immersed boundary method applied to the flow around a forced oscillating cylinder, J. Phys. Conf. Ser. 296 (2011) 12011. doi:10.1088/1742-6596/296/1/012011.

[44] J.I. Lee, L. Hu, P. Saha, M.S. Kazimi, Numeric al analysis of thermal striping induced high cycle thermal fatigue in a mixing tee, Nucl. Eng. Des. 239 (2009) 833-839. doi:10.1016/j.nucengdes.2008.06.014.

[45] J. Andreopoulos, On the structure of jets in a crossflow, J. Fluid Mech. 157 (1985) 163. doi:10.1017/S0022112085002348.

[46] A. Hoda, S. Acharya, Predictions of a Film Coolant Jet in Crossflow With Different Turbulence Models, J. Turbomach. 122 (2000) 558. doi:10.1115/1.1302322.

[47] I. Tiselj, J. Oder, L. Cizelj, Double-sided cooling of heated slab: Conjugate heat transfer DNS, Int. J. Heat Mass Transf. 66 (2013) 781-790. 
doi:10.1016/j.ijheatmasstransfer.2013.07.076.

[48] N. Kasagi, Y. Tomita, A. Kuroda, Direct Numerical Simulation of Passive Scalar Field in a Turbulent Channel Flow, J. Heat Transfer. 114 (1992) 598. doi:10.1115/1.2911323.

[49] D.A. Donzis, K.R. Sreenivasan, P.K. Yeung, Scalar dissipation rate and dissipative anomaly in isotropic turbulence, J. Fluid Mech. 532 (2005) 199-216. doi:10.1017/S0022112005004039.

[50] Z. Warhaft, Passive Scalars in Turbulent Flows, Annu. Rev. Fluid Mech. 32 (2000) 203-240. doi:10.1146/annurev.fluid.32.1.203.

[51] S.B. Pope, Turbulent Flows, Cambridge University Press, Cambridge, 2000. doi:10.1017/CBO9780511840531.

[52] H. Tennekes, J.L. Lumley, A First Course in Turbulence, MIT Press, 1972.

[53] Z. Warhaft, J.L. Lumley, An experimental study of the decay of temperature fluctuations in grid-generated turbulence, J. Fluid Mech. 88 (1978) 659. doi:10.1017/S0022112078002335.

[54] P.K. Yeung, B. Sawford, Random-sweeping hypothesis for passive scalars in isotropic turbulence, J. Fluid Mech. 459 (2002) 129-138. doi:10.1017/S0022112002008248.

[55] P.K. Yeung, S. Xu, K.R. Sreenivasan, Schmidt number effects on turbulent transport with uniform mean scalar gradient, Phys. Fluids. 14 (2002) 4178-4191. doi:10.1063/1.1517298. 